\title{
Perturbation and restoration of the fathead minnow gut microbiome after low-level triclosan exposure
}

Adrienne B Narrowe ${ }^{1}$, Munira Albuthi-Lantz ${ }^{1}$, Erin P Smith ${ }^{1,2,3}$, Kimberly J Bower ${ }^{1}$, Timberley M Roane ${ }^{1}$, Alan M Vajda ${ }^{1}$ and Christopher S Miller ${ }^{{ }^{*}}$

\begin{abstract}
Background: Triclosan is a widely used antimicrobial compound and emerging environmental contaminant. Although the role of the gut microbiome in health and disease is increasingly well established, the interaction between environmental contaminants and host microbiome is largely unexplored, with unknown consequences for host health. This study examined the effects of low, environmentally relevant levels of triclosan exposure on the fish gut microbiome. Developing fathead minnows (Pimephales promelas) were exposed to two low levels of triclosan over a 7-day exposure. Fish gastrointestinal tracts from exposed and control fish were harvested at four time points: immediately preceding and following the 7-day exposure and after 1 and 2 weeks of depuration.

Results: A total of 103 fish gut bacterial communities were characterized by high-throughput sequencing and analysis of the V3-V4 region of the 16S rRNA gene. By measures of both alpha and beta diversity, gut microbial communities were significantly differentiated by exposure history immediately following triclosan exposure. After 2 weeks of depuration, these differences disappear. Independent of exposure history, communities were also significantly structured by time. This first detailed census of the fathead minnow gut microbiome shows a bacterial community that is similar in composition to those of zebrafish and other freshwater fish. Among the triclosan-resilient members of this host-associated community are taxa associated with denitrification in wastewater treatment, taxa potentially able to degrade triclosan, and taxa from an unstudied host-associated candidate division.

Conclusions: The fathead minnow gut microbiome is rapidly and significantly altered by exposure to low, environmentally relevant levels of triclosan, yet largely recovers from this short-term perturbation over an equivalently brief time span. These results suggest that even low-level environmental exposure to a common antimicrobial compound can induce significant short-term changes to the gut microbiome, followed by restoration, demonstrating both the sensitivity and resilience of the gut flora to challenges by environmental toxicants. This short-term disruption in a developing organism may have important long-term consequences for host health. The identification of multiple taxa not often reported in the fish gut suggests that microbial nitrogen metabolism in the fish gut may be more complex than previously appreciated.
\end{abstract}

Keywords: Triclosan, Fathead minnow, 16S, Gut microbiome, Denitrification

\footnotetext{
* Correspondence: chris.miller@ucdenver.edu

${ }^{1}$ Department of Integrative Biology, University of Colorado Denver,

Campus Box 171, PO Box 173364, Denver, CO 80217, USA

Full list of author information is available at the end of the article
} 


\section{Background}

The importance of host-associated gut microbiota to the normal development and overall health of the host organism is well-established and increasingly appreciated [1-3]. The gut microbiome has been shown to be both stable over the long-term [4] and vulnerable to disruption [5] which may have long-term implications for host health [6,7]. Studies on the chemical disruption of hostassociated microbiota have generally been concerned with the clinical use of antibiotics $[6,8,9]$, the intentional exposure to personal care products [10], or have focused on the effect of antimicrobials on specific taxa [11]. Less well-characterized are the challenges to the healthy hostassociated microbiome from common contaminants, including antimicrobial compounds, particularly at low, but environmentally relevant levels. Even short-duration, low-concentration exposures may alter the gut flora during developmentally important windows.

One such emerging contaminant is triclosan (5-chloro2-(2,4-dichlorophenoxy)phenol), a chlorinated aromatic compound that has been used as an antimicrobial since the 1960s [12]. Triclosan has limited clinical application, but is frequently found as a component of personal care products and household products [13]. Triclosan is ubiquitous, enters the wastewater system, persists through the wastewater treatment process [14], and has been detected in surface waters at concentrations up to $2.3 \mu \mathrm{g} \mathrm{L} \mathrm{L}^{-1}$ [15]. Triclosan has been associated with adverse physiological and developmental outcomes [16-18], is photodegraded to produce dioxins [13], and is potentially an endocrine disruptor in fish [19]. Recently, the United States Food and Drug Administration has reopened discussion of the regulation of triclosan [20], and the state of Minnesota has banned the sale of consumer products containing this compound [21]. Despite this increased interest in triclosan, nothing is known about indirect effects on host health, either due to the triclosan-mediated alteration of the microbiome, or due to the microbially mediated transformation of triclosan.

We hypothesized that low, environmentally relevant levels of triclosan exposure are sufficient to disrupt the fish gut microbiome. To test this hypothesis, we exposed larval fathead minnows (Pimephales promelas) to two levels of triclosan (100 and 1,000 $\mathrm{ng} \mathrm{L}^{-1}$ ) in a controlled laboratory experiment and used high-throughput $16 \mathrm{~S}$ rDNA sequencing to profile gut microbial communities before exposure, immediately following an acute exposure window, and after depuration. We also sought to provide a census of the gut bacterial community of the untreated juvenile fathead minnow, an important environmental toxicology model organism [22].

\section{Results}

16S V3-V4 variable region sequencing of fish gut microbiomes

Using 16S hypervariable region sequencing, we characterized gut microbiomes of fathead minnows in a controlled triclosan exposure experiment. Fish were divided into four exposure groups representing low and high triclosan exposure levels and unexposed and solvent-only control groups (Figure 1). A total of 103 microbiomes were characterized, including eight baseline samples (day 0), samples collected immediately after a 7-day exposure window (day 7), and during a depuration period (day 14 and day 21). At each time point, eight fish were collected for each exposure group. Due to a failure in DNA extraction, one fish from the day 14 low exposure group was not sequenced. An additional five samples were sequenced: one technical replicate (repeat PCR, sequencing, and analysis), one mock community constructed of ten bacteria and archaea (Table 1), and three 'spike-in' control samples consisting of a combination of sample gDNA and mock community DNA. Dual-indexed $2 \times 250$ bp Illumina MiSeq sequencing of $16 \mathrm{~S}$ rDNA

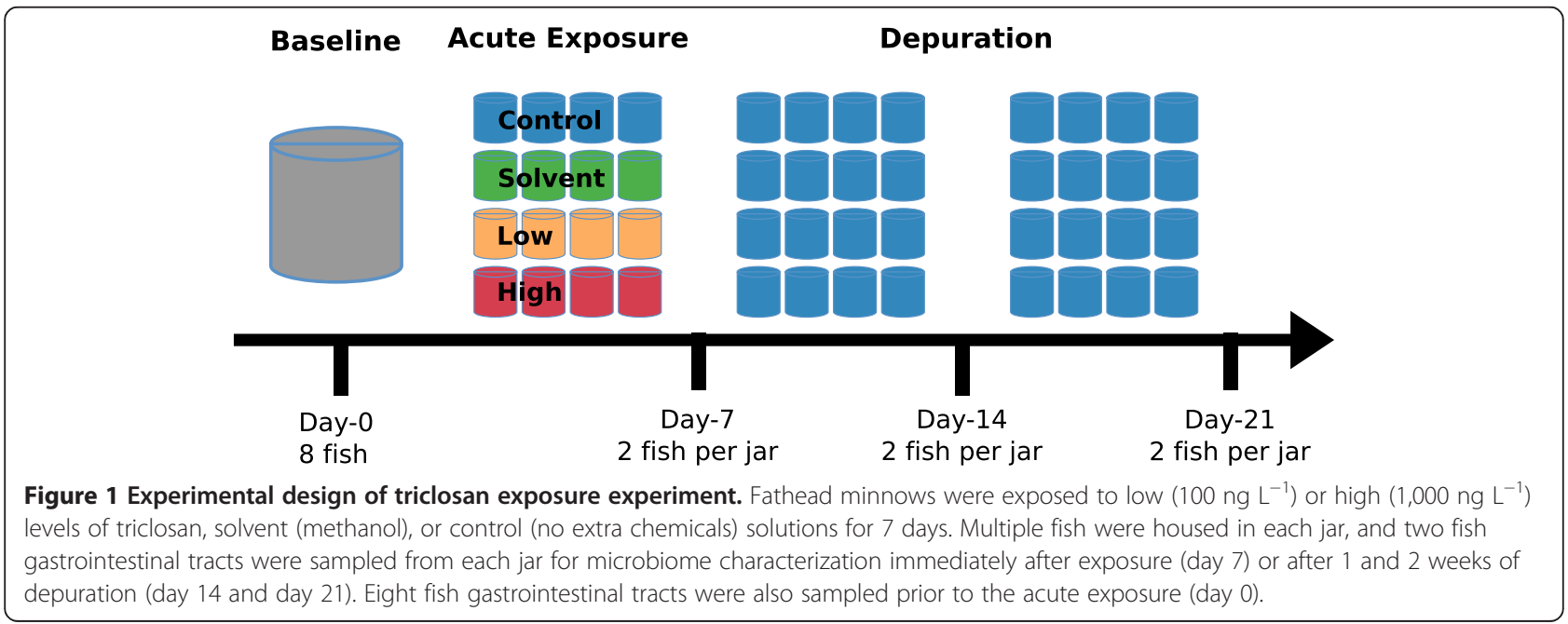


Table 1 Composition and accuracy of identification of mock community 16S rDNA sequences

\begin{tabular}{|c|c|c|}
\hline OTU ID & Pipeline assigned taxonomy & Mock community ID (ATCC or GenBank) \\
\hline OTU13 & $\begin{array}{l}\text { k__Bacteria; P_Proteobacteria; c__Gammaproteobacteria; O_Alteromonadales; } \\
\text { f__Shewanellaceae; g__Shewanella; s_ }\end{array}$ & Shewanella amazonenesis SB2B ATCC: BAA-1098 \\
\hline OTU32 & $\begin{array}{l}\text { k_Bacteria; P_Firmicutes; C__Bacilli; O_Lactobacillales; f_Lactobacillaceae; } \\
\text { g__Pediococcus; s__ }\end{array}$ & Pediococcus pentosaceus ATCC: 25745 \\
\hline OTU27 & k_Bacteria; P_Firmicutes; c__Bacilli; o__Bacillales; f_Bacillaceae; g_Bacillus; s__ & $\begin{array}{l}\text { Uncharacterized Bacillus isolate GenBank ID: } \\
\text { KP025972 }\end{array}$ \\
\hline OTU21 & $\begin{array}{l}\text { k_Bacteria; P_Firmicutes; C__Bacilli; O_Lactobacillales; f_Lactobacillaceae; } \\
\text { g__Lactobacillus; s_zeae }\end{array}$ & Lactobacillus casei ATCC: 334 \\
\hline OTU20 & $\begin{array}{l}\text { k_Bacteria; P_Firmicutes; c__Bacilli; o__Lactobacillales; f__Streptococcaceae; } \\
\text { g__Lactococcus; S__ }\end{array}$ & $\begin{array}{l}\text { Lactococcus lactis SK11 ATCC: BAA-493 } \\
\text { Lactococcus lactis IL } 403\end{array}$ \\
\hline OTU26 & $\begin{array}{l}\text { k_Bacteria; P_Firmicutes; c__Bacilli; O_Lactobacillales; f_Lactobacillaceae; } \\
\text { g__Lactobacillus; s__brevis }\end{array}$ & Lactobacillus brevis ATCC: 367 \\
\hline OTU30 & $\begin{array}{l}\text { k__Bacteria; P__Proteobacteria; C__Gammaproteobacteria; O_Enterobacteriales; } \\
\text { f__Enterobacteriaceae; g__ s__ }\end{array}$ & Escherichia coli K12 GenBank ID: U00096 \\
\hline OTU39 & k_Bacteria; P__Actinobacteria; c_Actinobacteria; o__Actinomycetales; f_; g__; s_ & Acidothermus cellulolyticus ATCC: 43068 \\
\hline OTU294 & $\begin{array}{l}\text { k__Bacteria; P__Proteobacteria; c__Gammaproteobacteria; O_Enterobacteriales; } \\
\text { f__Enterobacteriaceae; g__ s__ }\end{array}$ & Escherichia coli K12 GenBank ID: U00096 \\
\hline OTU102 & $\begin{array}{l}\text { k__Archaea; P_Euryarchaeota; c__Halobacteria; O_Halobacteriales; } \\
\text { f__Halobacteriaceae; g__Halobacterium; s__ }\end{array}$ & Halobacterium salinarum NRC-1 ATCC: 700922 \\
\hline
\end{tabular}

region V3-V4 amplicons from all 108 fish gut and technical samples produced 17,068,840 valid read pairs (Additional file 1: Table S1). All samples sequenced were included in all subsequent analyses. After merging reads, operational taxonomic unit (OTU) picking at 97\% identity, and removal of chimeric sequences (see the 'Methods' section), we identified 695 OTUs, 10 of which corresponded to the 10 organisms spiked in as members of a mock community. These 695 OTUs were represented by $11,118,352$ merged read pairs. Of these 695 OTUs, 94 (13.5\%) passed our filtering procedure and accounted for 9,012,689 merged read pairs $(81.1 \%$ of pre-filtering reads). To confirm that our strict filtering procedure did not alter the conclusions presented here, we also performed all analyses presented below on the unfiltered OTU table and found similar community composition and dynamics for all time and exposure conditions (Additional file 2: Figure S2 and Additional file 3: Figure S3).

\section{V3-V4 sequencing of fish samples is accurate and technically reproducible}

In order to assess the accuracy and reproducibility of V3-V4 hypervariable region library preparation and sequencing, we sequenced one mock community sample, three mock community spike-in control samples, and one replicate of sample D0C9. Community membership and abundance were highly correlated across replicate samples (Pearson $r=0.99$ between D0C9 replicates; Additional file 4: Figure S4). We used the mock community sample to guide quality control and filtering of lowlevel contaminants and spurious OTUs. Our pipeline identified all ten expected mock community V3-V4 sequences at $100 \%$ identity (Table 1; Additional file 4: Figure S4) with the following exceptions: the two subspecies of Lactococcus lactis were 100\% identical over the V3-V4 region surveyed and were collapsed together into OTU20. Escherichia coli K12 was represented by two OTUs. The E. coli K12 genome contains seven copies of the 16S rRNA gene, which are identical over the V3-V4 region. The second OTU assigned to E. coli was substantially less abundant (OTU294: mock community relative abundance 0.005) than the expected OTU (OTU30: mock community relative abundance 0.063 ) and may represent a contaminant or variant in the culture from which the DNA was acquired.

Our pipeline assigned 37 OTUs to our mock community sample. Of the reads from this sample, $99.89 \%$ were assigned to one of the ten expected mock community sequences, which were also the most abundant OTUs. The remaining 27 OTUs accounted for a total of only 103 reads (0.11\%; Additional file 4: Figure S4). These 27 OTUs were all found within the fish samples. Although we cannot rule out the unlikely possibility of miscalled multiplexing indices falsely placing reads into the wrong sample, these 27 OTUs are presumed to be low-level PCR cross-contamination occurring during sequencing library preparation, reflecting the sensitivity inherent to deep DNA sequencing. The most abundant non-mock community OTU found in the mock community sample was OTU1, a member of the family Aeromonadaceae. OTU1 represented $32 \%$ of all reads in the unfiltered OTU table and was the most abundant OTU in the study. Within the mock community, this OTU accounted for 
only $0.03 \%$ of reads; this abundance level was used as the basis for study-wide OTU filtering (see the 'Methods' section and Additional file 4: Figure S4). If present in the experimental samples, the mock community OTUs were successfully filtered out as contaminants. In the spike-in samples, the few remaining non-filtered reads (mean $n=263$ ) closely reconstructed the expected D0C9 community (Additional file 4: Figure S4; mean pairwise Pearson $r=0.85$ ). Thus, we proceeded to estimate fish gut microbial community composition under the assumption that any observed inter-individual differences were not primarily due to technical artifacts.

\section{The fathead minnow gut microbiome resembles that of other freshwater fish}

To our knowledge, we present here the first deep sequencing census of the gut microbiome of the fathead minnow, a common model organism in environmental toxicology studies. The eight co-housed baseline day 0 samples show low inter-individual variation in microbial community composition (mean pairwise Pearson $r=0.80$ ), as estimated based on the relative abundance of $16 \mathrm{~S}$ rRNA gene sequences observed. Among the most abundant taxa inferred in the fathead minnow gut are many bacteria previously observed in other freshwater fish [23-27]: Aeromonadaceae (mean abundance: $8.5 \%$ baseline, $57.5 \%$ day 7 to 21 control), Shewanellaceae (mean abundance: $2.2 \%$ baseline, 9.1\% day 7 to 21 control), as well as Flavobacteriaceae, Fusobacteriaceae, Deefgea, Pseudomonadaceae, Plesiomonas, and Cetobacterium. Some of these taxa are included in the set of 20 OTUs that were present across at least 95\% of the 103 sampled fish across all time points and exposures, representing a potential fathead minnow 'core' microbiome (Table 2; Additional file 5: Figure S5). Relative abundances of many of these core OTUs change with fish development during the course of the experiment in the unexposed controls. For example, OTU10 (Deefgea), OTU11 (Pseudomonas), and OTU6 (Rhodobacter) appear to increase in relative abundance from days 7 to 21, while OTU9 (Pseudoalteromonadaceae), OTU4 (CK-1C4-19), and OTU482 (Aeromonadaceae) decrease. In addition to considering variation at different developmental stages, definitive identification of a core fathead minnow microbiome would require sampling from a variety of habitats and genotypes and in laboratory settings may be affected by environmental conditions such as water chemistry, water recirculation, and diet.

\section{Gut community composition changes over time}

We calculated measures of alpha (within sample) diversity and beta (between samples) diversity among gut microbiomes. Alpha diversity, as measured by the Shannon diversity index, decreased sharply for all exposure groups from baseline (arrival from rearing facility) to

Table 2 Twenty OTUs form a study-wide core fathead minnow gut microbiome

\begin{tabular}{|c|c|c|c|}
\hline OTU IDs & Taxonomic assignment & Phylum & Mean relative abundance \\
\hline OTU2 & Acinetobacter (genus) & Proteobacteria & $0.053 \pm 0.057$ \\
\hline OTU46 & Acinetobacter (genus) & Proteobacteria & $0.017 \pm 0.02$ \\
\hline OTU392 & Acinetobacter johnsonii (species) & Proteobacteria & $0.004 \pm 0.006$ \\
\hline OTU1 & Aeromonadaceae (family) & Proteobacteria & $0.37 \pm 0.18$ \\
\hline OTU482 & Aeromonadaceae (family) & Proteobacteria & $0.08 \pm 0.11$ \\
\hline OTU600 & Aeromonadaceae (family) & Proteobacteria & $0.02 \pm 0.09$ \\
\hline OTU14 & Bacteroidaceae (family) & Bacteroidetes & $0.007 \pm 0.012$ \\
\hline OTU16 & Betaproteobacteria (class) & Proteobacteria & $0.008 \pm 0.013$ \\
\hline OTU8 & Cetobacterium somerae (species) & Fusobacteria & $0.03 \pm 0.04$ \\
\hline OTU4 & CK-1C4-19 (candidate division) & CK-1C4-19 & $0.03 \pm 0.06$ \\
\hline OTU10 & Deefgea (genus) & Proteobacteria & $0.02 \pm 0.04$ \\
\hline OTU12 & Flavobacterium (genus) & Bacteroidetes & $0.01 \pm 0.01$ \\
\hline OTU18 & Fusobacteriales (order) & Fusobacteria & $0.005 \pm 0.007$ \\
\hline OTU9 & Pseudoalteromonadaceae (family) & Proteobacteria & $0.03 \pm 0.05$ \\
\hline OTU11 & Pseudomonas (genus) & Proteobacteria & $0.02 \pm 0.03$ \\
\hline OTU40 & Pseudomonas alcaligenes (species) & Proteobacteria & $0.002 \pm 0.003$ \\
\hline OTU6 & Rhodobacter (genus) & Proteobacteria & $0.03 \pm 0.06$ \\
\hline OTU7 & Shewanella (genus) & Proteobacteria & $0.1 \pm 0.06$ \\
\hline OTU760 & Shewanella (genus) & Proteobacteria & $0.001 \pm 0.001$ \\
\hline OTU5 & Verrucomicrobiaceae (family) & Verrucomicrobia & $0.03 \pm 0.04$ \\
\hline
\end{tabular}

Mean relative abundance from 103 samples collected across four time points and five exposure categories. 
day 7 (Figure 2). Two-way ANOVA considering the effects of time point and triclosan exposure group on mean Shannon diversity index indicated no interaction effect $(p=0.67)$, so we considered each main effect separately. Using Tukey's honest significant difference (HSD) test for multiple comparisons (Figure 2), the baseline samples differ significantly from all other time points $(P \leq 0.012)$. At day 7 only, the control samples differed significantly from both the low and high samples $(P=0.026, P=0.001)$ and the solvent samples differed from the high samples $(P=0.017)$ with alpha diversity increasing in association with triclosan exposure. Within the later time points, there was no significant difference in the mean Shannon diversity index associated with triclosan exposure. The same analyses applied to the unfiltered OTU table produced the same set of significant pairwise differences in alpha diversity.

For beta diversity, a weighted UniFrac distance matrix [28] among all samples was calculated as input to principle coordinates analysis (PCoA) and as the basis of statistical tests described below. We used the weighted UniFrac metric, as our experimental design was of a closed microbial system, using sterilized materials including water and commercially prepared food (see the 'Methods' section): we expect changes in diversity among samples to be driven more by changes in relative abundance than by changes in community membership due to immigration. PCoA of weighted UniFrac distances separated samples primarily according to time point, with $68.8 \%$ of variation among samples explained by the first two axes (Figure 3a). PERMANOVA analysis [29] showed significant effects of both time $(F=18.4, d f=2, P \leq 0.001)$ and triclosan exposure $(F=13.6, d f=4, P \leq 0.001)$, with no interaction effect between the two factors $(F=1.2, d f=6, P=0.238)$. Baseline samples were most distant from all other samples. To ensure that the time and exposure effects across the entire experiment were not solely due to the divergence of baseline samples from all other samples, we repeated the principle coordinates analysis and the PERMANOVA after removing the baseline samples (Figure $3 \mathrm{~b}$ ). Again, the samples clustered according to time $(F=20.4924, d f=2$, $P \leq 0.001)$ and triclosan exposure $(F=2.2077, d f=3$, $P=0.024)$, with no significant interaction effect $(F=1.3072$, $d f=6, P \leq 0.170)$. This time signal is not an artifact of the combination of distance and ordination methods. The use of non-phylogenetically based distance methods (Canberra,

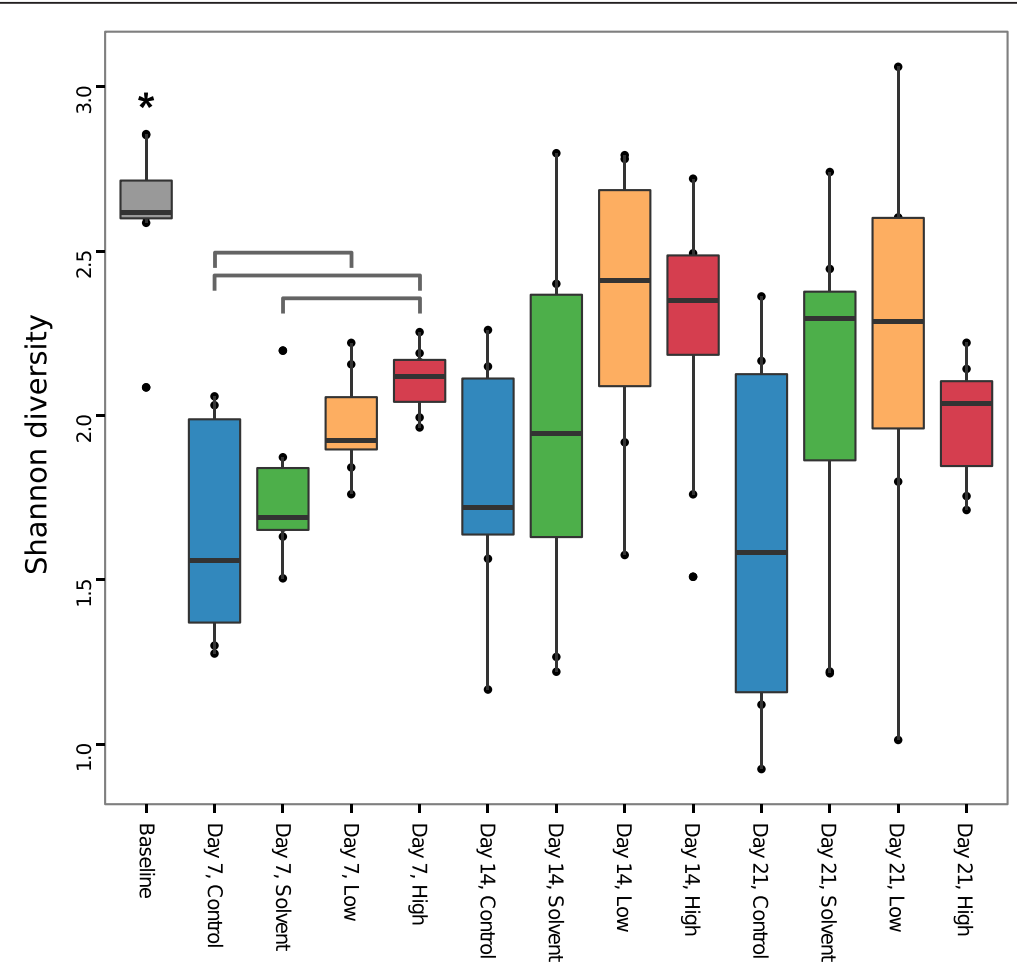

Figure 2 Alpha diversity pattern with time and triclosan exposure. Shannon diversity measures are plotted as interquartile range with median for each triclosan exposure class and time point grouping. There is an initial drop in alpha diversity at the beginning of the study. Baseline samples are significantly more diverse than all other time points (denoted by asterisk; Tukey's HSD, adjusted $P \leq 0.05$ ). Within day 7 , square brackets denote that the control samples differ significantly from low exposure $(P=0.026$; estimated difference in mean: $0.327 ; 95 \% \mathrm{Cl}$ : 0.031 to 0.623 ) and high exposure samples ( $P=0.001$; estimated difference in mean: $0.464 ; 95 \% \mathrm{Cl}: 0.167$ to 0.760$)$ and the solvent samples differ significantly from the high exposure samples ( $P=0.017$; estimated difference in mean: $-0.348 ; 95 \% \mathrm{Cl}:-0.644$ to -0.052$)$ For each boxplot, $n=8$ except day 14 , low $(n=7)$. 

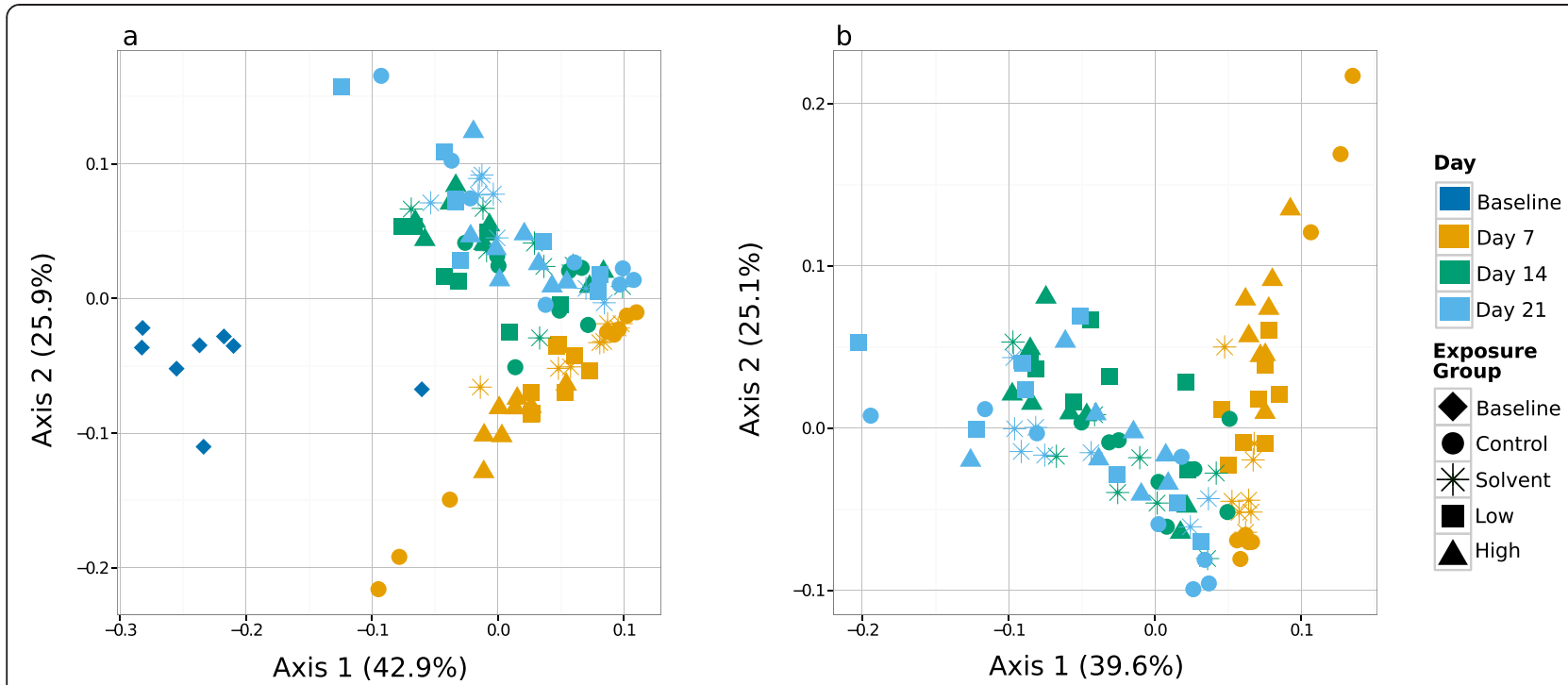

Figure 3 Fathead minnow gut microbial communities are dynamic over time. Principle coordinates analyses of weighted UniFrac distances show that gut communities cluster by time point. (a) Baseline samples (day 0) are distinct from samples collected at later time points. Samples from day 7 have a unique community structure that differentiates them from days 14 and 21 samples, which are largely indistinguishable from each other. (b) PCoA plot of weighted UniFrac distances calculated excluding the highly divergent baseline samples shows a similar divergence of day 7 samples from day 14 and day 21 samples.

Morisita) [30] and alternate ordinations generated the same groupings of samples, organized most strongly by time (Additional file 6: Figure S6).

\section{Environmental exposure to triclosan alters fish gut microbial community composition}

In order to test whether environmental exposure to triclosan alters fathead minnow gut microbiome composition independently of time, we evaluated each time point separately. Immediately following the triclosan exposure period (day 7), samples cluster visibly by triclosan exposure (Figure 4a). A secondary cluster of control samples (D7C32, D7C41, D7C42) is driven by the retention of OTUs from the Verrucomicrobiaceae and CK-1C419 which were present in the baseline (day 0) samples, but reduced in the other day 7 control samples (Additional file 7: Figure S1). At day 7, microbiomes exposed to high levels of triclosan were, on average, more distant from control microbiomes than those exposed to low levels of triclosan. Visual clustering by triclosan exposure disappears over the 2-week post-exposure depuration. Although samples are non-randomly distributed by

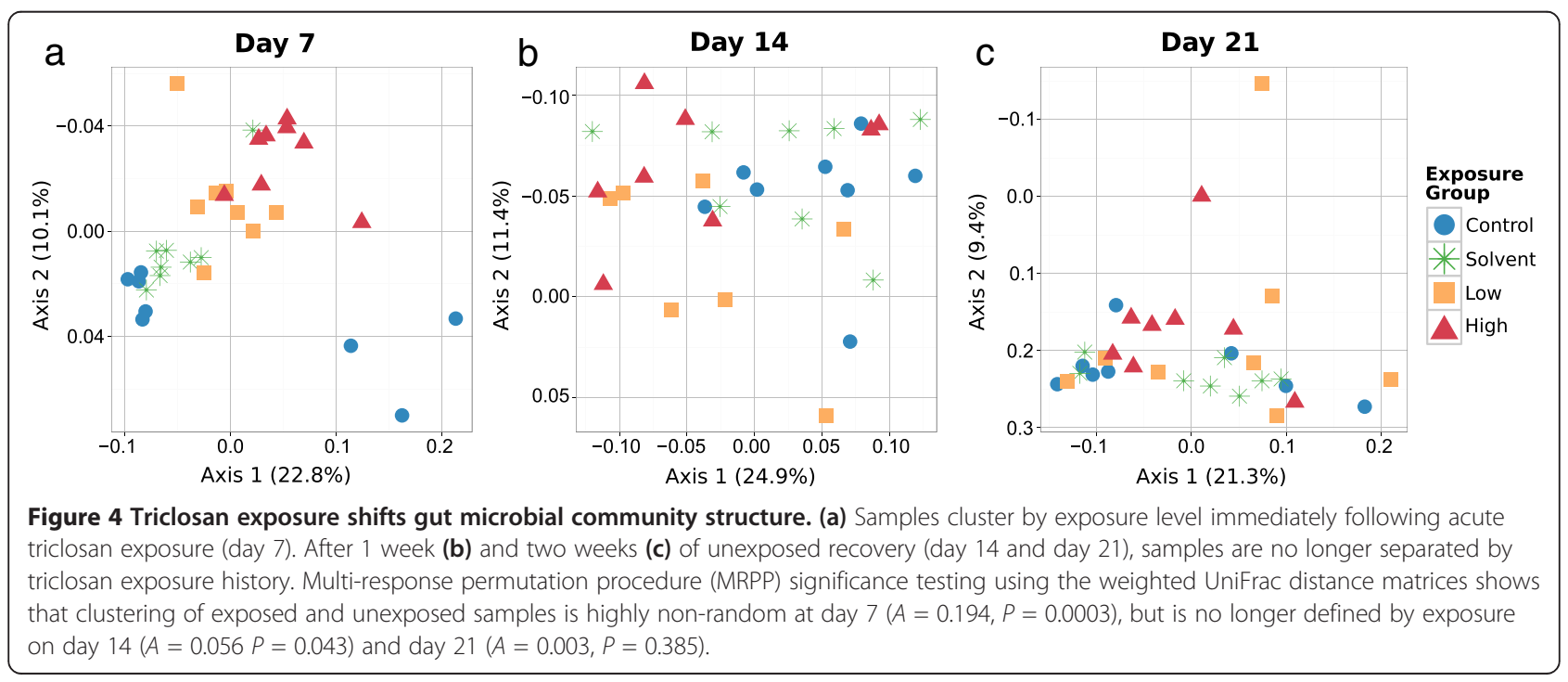


exposure status at day 14 (Figure 4b), at 2 weeks following exposure (day 21), microbiomes are indistinguishable by exposure type (Figure 4c). We confirmed the significance of the clustering by exposure type using the multi-response permutation procedure (MRPP) [31] on the weighted UniFrac distance matrix. This procedure compares the average observed withinexposure group distance against the within-group distance of randomly permuted groups. The $P$ values associated with exposure effect increase from 0.0003 at day 7 to 0.043 at day 14 to 0.385 at day 21 (Figure 4). The use of Canberra and Morisita distance metrics as noted above confirmed (both via ordination and via MRPP) that the observed clustering by triclosan exposure status at day 7 was not an artifact of the choice of distance and ordination metrics (Additional file 8: Figure S7).

\section{Differential response to time and triclosan exposure can be seen for individual taxa}

Examining the community at the order level (Figure 5) identifies specific taxa that contribute to temporal and triclosan exposure-associated changes in overall microbial community structure (Figures 2, 3, and 4). Housing conditions were designed to minimize the chance that changes to microbial community membership could result from newly introduced taxa after the initiation of this study, as all housing materials and water were sterilized. There were no new bacterial orders present in days 7 to 21 that were not present in at least one baseline

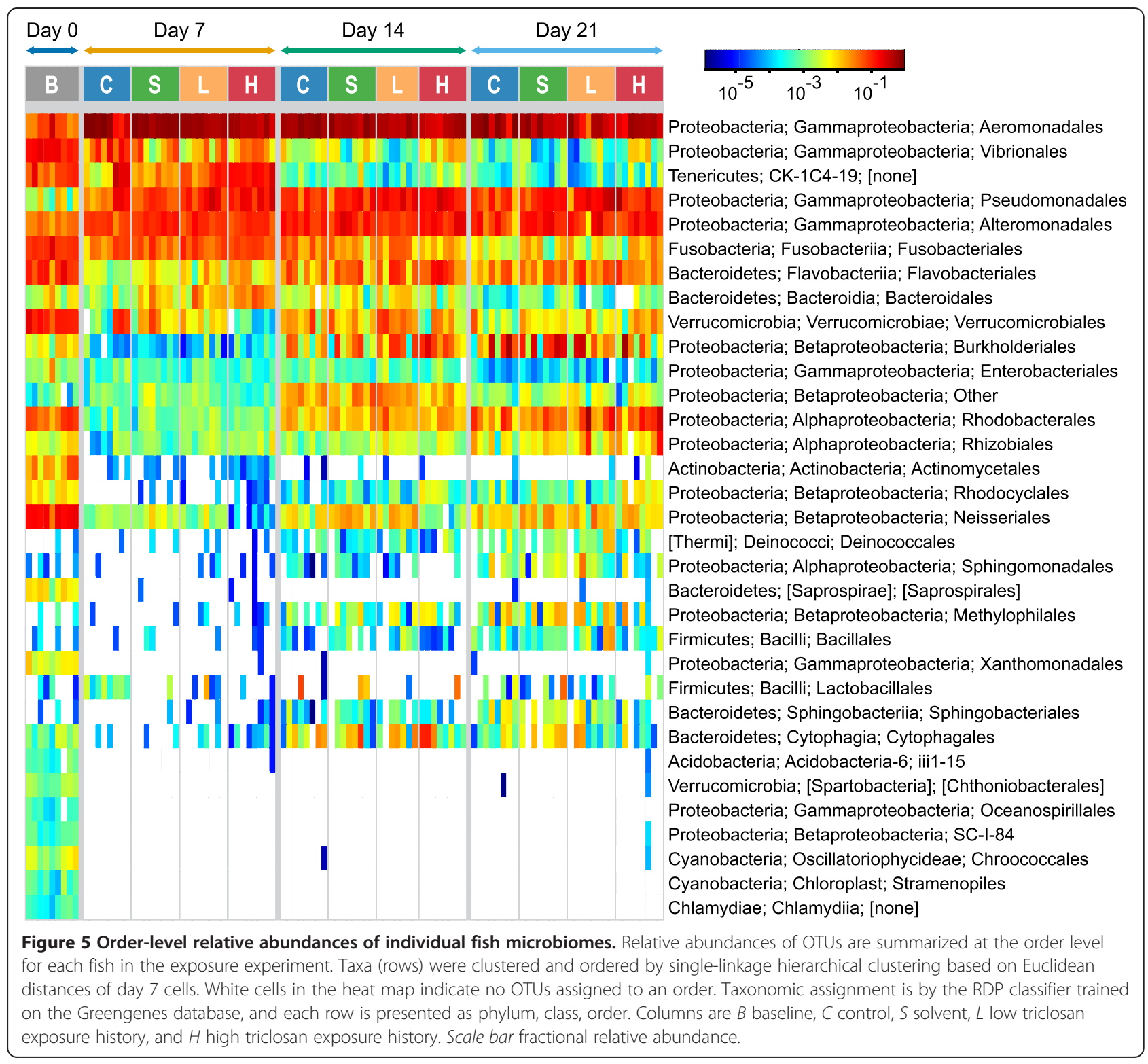


(day 0) sample. At the OTU level, there were five OTUs present in days 7 to 21 that were never detected in a baseline sample, though each of these OTUs had at least one other closely related OTU in at least one baseline sample at the family or genus level. However, many orders present in day 0 were lost or fell below the level of detection at subsequent sampling time points. Below the order level, 28 individual OTUs were significantly differentially abundant (DESeq2 [32]; adjusted $P<=0.005$ ) between unexposed samples (control and solvent) and exposed (low and high) samples at day 7 (Figure 6 and Additional file 9: Table S2). These OTUs include but are not limited to members of Cetobacterium, Methylobacterium, Flavobacterium, Methylotenera, Hydrogenophaga, and CK-1C4-19. At day 14, only two OTUs were differentially abundant and by day 21 , no OTUs were significantly more abundant when comparing these two classes. Considering the effects of triclosan independent of solvent exposure, at day 14 and day 21, Hydrogenophaga OTU624 was increased in relative abundance in high-triclosan exposed samples relative to solvent control samples and, at day 21, Thauera OTU37 was increased in relative abundance in high samples relative to solvent samples (Figure 7 and Additional file 10: Table S3 and Additional file 11: Table S4).

\section{Fathead minnow Gl tracts contain highly abundant members of the poorly characterized candidate division CK-1C4-19}

Within the fathead minnow gut bacterial communities, we identified a highly abundant taxon, CK-1C4-19, that is classified as either a member of the phylum Tenericutes [33] or a candidate division at the phylum level [34]. OTUs from this taxon have been previously identified as members of the zebrafish gut microbiome [23], and a relatively small number of sequences exist in $16 \mathrm{~S}$ rDNA databases, with annotated environmental and host-associated habitats including other cyprinid fishes, ants, lobster, catfish, sediments, and anaerobic digesters. The two CK-1C419 OTUs observed in fathead minnow guts most closely resemble those reported from other cyprinid fishes (Figure 8). This taxon was abundant in all baseline samples, ranging from $2.2 \%$ to $22 \%$ relative abundance, and was present in all day 7 samples. In general, CK-1C4-19 increased in relative abundance with triclosan exposure, with relative abundance ranging from $1.6 \%$ to $14.6 \%$ in the day 7 low samples and $10.9 \%$ to $18.3 \%$ in the day 7 high samples (Figure 8, inset). By comparison, CK-1C4-19 was present as $0.2 \%$ to $36 \%$ of day 7 control samples and $1.2 \%$ to $5.4 \%$ of day 7 solvent samples. As this is a change in relative abundance, we cannot rule out that the apparent

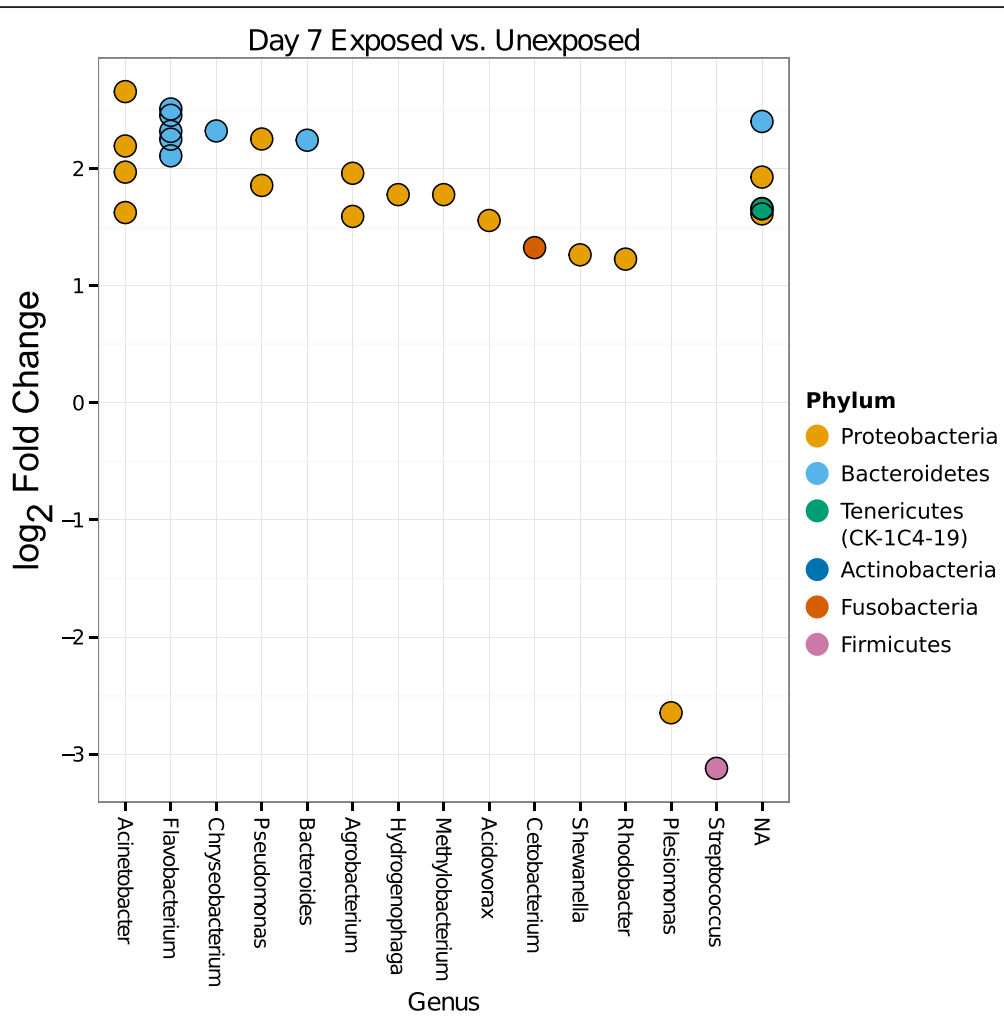

Figure 6 Significant changes in abundance of selected gut microbiome OTUs following triclosan exposure. OTUs were identified as significantly differentially abundant (adjusted $P \leq 0.005$ ) by exposure status (exposed [high and low] vs. unexposed [control and solvent]) at day 7 via DESeq2 [32]. Genus-level assignments are presented where available. 


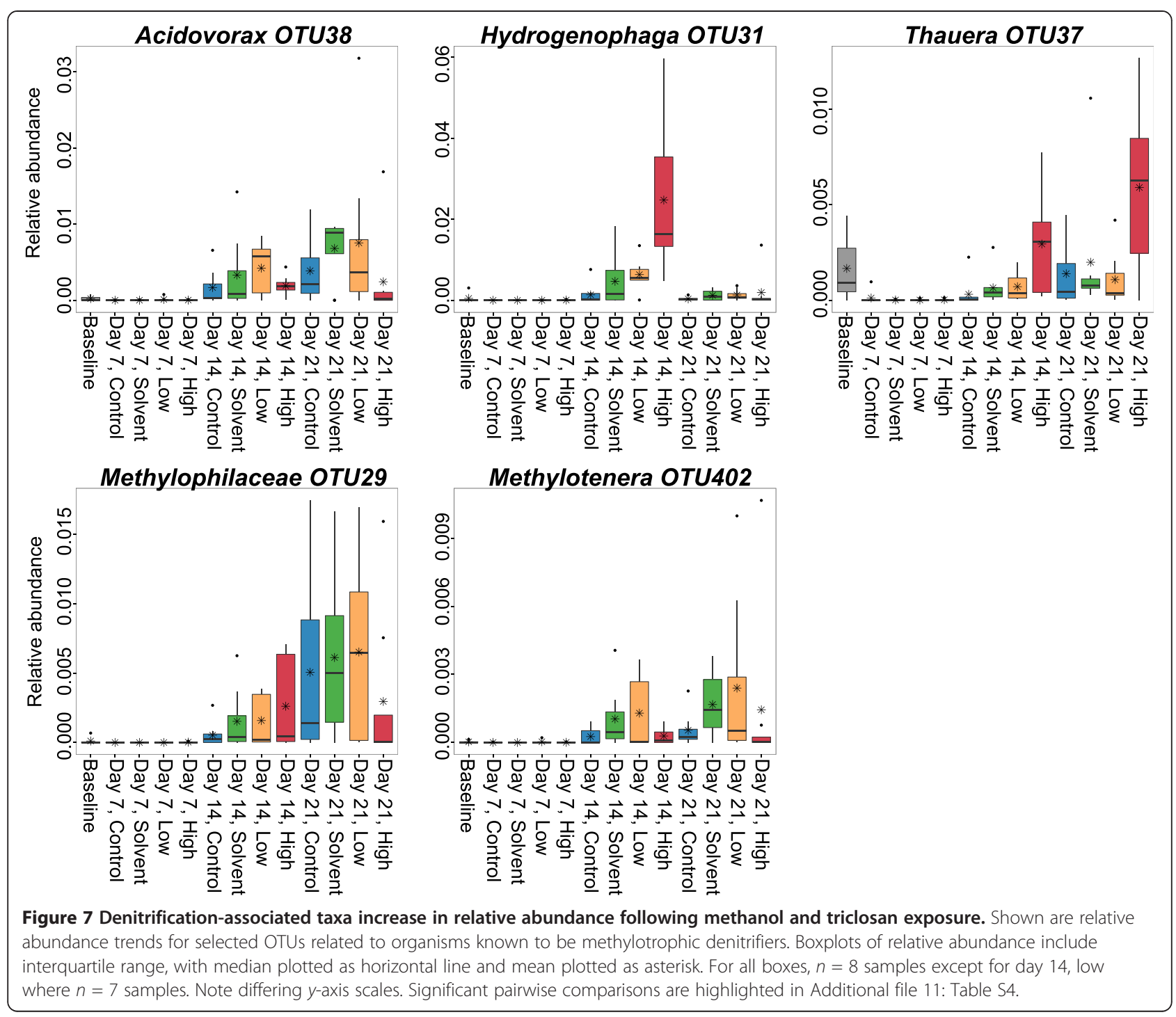

increase may reflect a decrease in abundance of other taxa, with CK-1C4-19 remaining unchanged. Despite a dramatic drop in abundance after day 7, CK-1C4-19 was present in all but one of the day 14 samples and was found in all but three of the day 21 samples.

\section{Discussion}

Despite the low but environmentally relevant [15] levels (100 to $1,000 \mathrm{ng} \mathrm{L}^{-1}$ ) used here, acute triclosan exposure is sufficient to disrupt fish gut bacterial community structure (Figure 4). In addition, fish microbiomes from the two exposure levels are visually separated in the PCoA, which may indicate a dosage effect. Triclosan targets the final step of the bacterial fatty acid synthesis pathway, inhibiting the active site of the enoyl-acyl reductase FabI [35]. Insensitivity to triclosan results from mutations in the $f a b I$ gene $[35,36]$, FabI overexpression [35], the expression of alternate Fab proteins such as
FabK, FabL, and FabV [37-39], or through efflux [40]. Initially described in Vibrio sp., FabV is reported to confer greater resistance to triclosan than mutant forms of FabI [39]. FabV has also been identified in other Gramnegative bacteria including members of the Aeromonas, Shewanella, and Pseudomonas genera, organisms highly abundant in freshwater fish gut microbiomes. The prevalence of fish gut bacterial species maintaining variant Fab genes may explain the stable presence of certain taxa across our study, such as Aeromonadaceae and Shewanella. In addition, triclosan may be either bactericidal or bacteriostatic depending on concentration [41]. Finally, overall microbial community structure may contribute to differences in triclosan bioavailability and effect, if the community contains members that are resistant to or are able to metabolize triclosan.

Although order-level (Figure 5) or genus-level (Additional file 7: Figure S1) patterns of changes in abundance among 


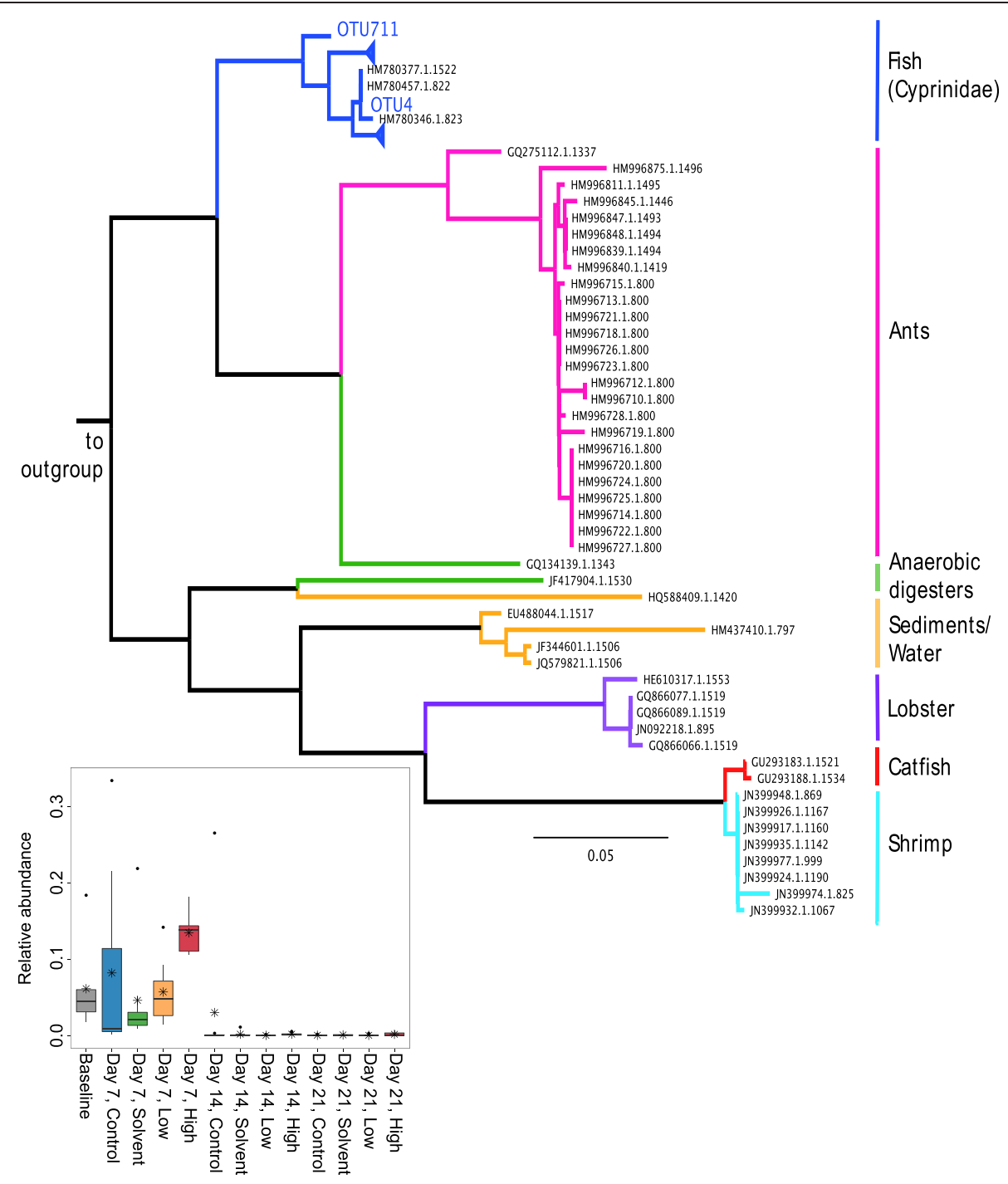

Figure 8 CK-1C4-19 taxa are phylogenetically distributed by host and habitat. The V3-V4 regions from publically available 16S rDNA sequences assigned to the candidate division CK-1C4-19 were aligned with CK-1C4-19 sequences found in the fathead minnow gut (OTU4, OTU711), and a phylogenetic tree was constructed. OTUs found in the fathead minnow gut are most closely related to sequences reported from other fish in the family Cyprinidae. Scale bar changes per site. Inset: relative abundances by time and treatment of the dominant CK-1C4-19 OTU4.

exposure groups were clearly visible, many interesting differential responses to triclosan at the OTU-level were obscured by summarizing at higher taxonomic levels. For example, while as an aggregate, all members of the order Pseudomonadales are highly abundant over the course of this study (Figure 5); the abundance patterns of the five Acinetobacter OTUs in this order suggest differing OTUlevel responses to environmental factors, including triclosan (Additional file 12: Figure S8). In addition to Acinetobacter, the 26 OTUs whose abundance increased at day 7 in association with triclosan exposure included Flavobacterium, Chryseobacterium, and Shewanella; all four genera contain a subset of species that are documented pathogens of fish and humans [42-45]. Another differentially abundant OTU was classified as candidate division CK-1C4-19, a rarely identified taxon previously documented within the zebrafish gut microbiota [23]. Repeated detection of CK-1C4-19 within the guts of closely related hosts despite geographical distance and its apparent insensitivity to triclosan (Figure 8) warrant further study examining the potential functional contribution to or dependence on its host.

Also included among the OTUs significantly increased in abundance with triclosan exposure were species of Methylobacterium, Hydrogenophaga, and Acidovorax (Figure 6). Methylotrophic proteobacteria such as these are commonly observed as members of denitrifying consortia in wastewater treatment plants $[46,47]$. These consortia can include Thauera and Methylotenera [47-49], other genera identified in this study. The microbial 
denitrification process in wastewater treatment is often facilitated by amendment with carbon substrates including methanol [50] which can be utilized by methylotrophic denitrifiers such as those identified here [51]. Over the course of this study, which used methanol for triclosan solubilization, OTUs identified as Hydrogenophaga, Methylophilaceae, Methylotenera, Thauera, and Acidovorax displayed a similar pattern of changes in relative abundance (Figure 7 and Additional file 11: Table S4). These OTUs were low in overall abundance at day 7 and became more prominent at later time points, particularly in triclosan-exposed and solventexposed samples. This pattern of increase in abundance suggests the possibility that methanol-enabled denitrification can occur within the fish gastrointestinal tract. Alternatively, because we did not measure changes in available nitrogen species in the water or characterize water microbial community changes over time, it is also possible that the combination of methanol (during the 7-day exposure) and the nitrogenous waste from the fish favored the growth of these organisms and that the measured increase in relative abundance of these taxa in the gut may in fact be due to a relative increase in transient bacteria that were sourced from the housing water. While these bacterial taxa were measured from the gut, this study did not differentiate between adherent or transient members of the gut microbial communities.

Hydrogenophaga spp. have been identified in association with amphibian hosts [52], but, to our knowledge, Thauera sp. have not been reported as members of vertebrate host-associated bacterial communities. Acidovorax OTUs have been noted in the trout gut [26] and also within the gut of the marine sea bream in a recent study that speculated on the possibilities of microbial denitrification occurring within the fish gut [53]. The presence of annamox bacterial taxa within fish guts has also been shown within the carp gut [54]. Host-associated denitrification and nitrous oxide production has been studied in freshwater invertebrates [55] and in earthworms [56], which also host Acidovorax species [57], but is less explored within vertebrate gut microbial communities, where removal of nitrate via reduction to ammonia rather than via denitrification is noted [58]. Thus, microbial nitrogen cycling pathways within the fish gut may be more diverse than have been reported within mammalian guts. Because of the obvious limitations to attempting to infer function from taxonomic information [59], functional studies are needed, including direct measurement of denitrification-associated gene expression within fish gut microbiomes and housing or habitat waters.

While most studies performed to identify triclosandegrading bacteria have focused on free-living rather than host-associated organisms, this catabolic ability has been demonstrated in bacteria across a wide variety of taxa [60], and it would not be surprising to find that members of the fish gut microbiome also possess the ability to metabolize or co-metabolize triclosan. Bacterial species related to those identified here have been associated with biodegradation of triclosan or other aromatic or halogenated compounds [47,61-66]. For example, OTU38 is a $100 \%$ BLAST match to a fully sequenced strain of Acidovorax that degrades poly-chlorinated biphenolic compounds [67], and Thauera has been specifically identified as a denitrifying organism with the potential to degrade aromatic compounds [68]. Thauera and Hydrogenophaga were differentially abundant at day 14 and/or day 21 in high samples with respect to solvent samples (Additional file 10: Table S3), suggesting that these organisms may derive some competitive benefit from even a short exposure to triclosan, independent of the presence of the methanol solvent. Microbial degradation of halogenated aromatics typically requires an aerobic environment [69]. Oxygen availability is likely to be variable over the length of the fish gastrointestinal tract, so sufficient oxygen may be present to support this process. If occurring in the gut, such microbially mediated processes could result in the direct intra-lumenal exposure to triclosan degradation products, including the lipophilic end product methyl-triclosan, which has been shown to bioaccumulate within fish [70].

Broadly, the differences in microbial community structure seen immediately following triclosan exposure (day 7) do not persist during depuration. By the conclusion of 2 weeks of recovery (day 21), as a whole, the communities cannot be distinguished on the basis of prior triclosan exposure, suggesting that most of the gut communities have returned to the same developmental path as those of the unexposed fish. Three samples from the triclosan-exposed groups remained distant from the main group of samples (Figure 4c), and in some cases, restoration of the initial community may take longer than our experimental window permits us to observe, or may never be complete, as has been shown for human microbiomes perturbed with antibiotic exposure [5]. The apparent increase in alpha diversity associated with triclosan exposure (Figure 2 ) is difficult to interpret, as short-term antibiotic exposure has been shown to decrease [5,6] or increase [6] diversity in other vertebrate guts.

In the case of low concentrations of triclosan, as used here, a bacteriostatic effect on most taxa, rather than a bactericidal one, could result in a near-complete restoration of community structure after depuration; however, even short-term disruption to the gut ecology, as demonstrated here, may be harmful to a developing host, with the potential for both immediate and long-ranging effects. Typical fish commensals such as Aeromonas, Deefgea, and Flavobacterium sp. can also be pathogens $[42,71,72]$ and may be held in check by less abundant, 
triclosan-sensitive members of the fish gut microbiome $[24,73]$. Thus, even a brief imbalance from acute exposure may precipitate opportunistic infections [74]. The fish gut microbiome is implicated in nutrient absorption and growth [75,76], so juvenile dysbiosis as a result of environmental toxicants may impact long-term fitness at the individual or population level in contaminated habitats. In surface water ecosystems, increased time (constant rather than acute) and intensity (concentration) of contaminant exposure could easily result in permanent alteration of host microbiome, with potential for ecosystemscale consequences.

Microbial communities of the fish gut are underexplored relative to the contribution of fish species to overall vertebrate diversity [77]. The fathead minnow ( $P$. promelas) is an important model organism for aquatic environmental toxicology [22] and is widely distributed in North America, and its developmental and reproductive response to environmental contaminants is wellcharacterized [78]. The gut microbiome described here adds to the limited catalog of fish microbiomes characterized by high-throughput sequencing [79]. Despite the temporal and triclosan exposure-associated differences in the microbial community structure, 20 of the 94 OTUs reported here constitute 'core' organisms present in $95 \%$ of the 103 samples sequenced (Table 2). These study-wide core OTUs include members of Aeromonadaceae, Bacteroidaceae, Shewanella, Pseudomonas, Deefgea, Acinetobacter, Flavobacterium, Cetobacterium, and others. Both technical differences (cultured vs. sequenced organisms, clones vs. short amplicons, choice of variable region sequenced, primer bias, sequencing depth, analysis) as well as differences related to the fish (age, diet, fresh caught vs. domesticated, water quality, husbandry, habitat) limit the ability to make direct comparisons of relative abundances across studies of other freshwater omnivorous fish gut microbiomes. Despite these caveats, these fathead minnow core microbiome OTUs are generally similar to the most common genera reported previously for zebrafish [23], the common carp [54] (both also family Cyprinidae), other freshwater omnivorous fish $[24,25,80]$, and, in a limited fashion, for the fathead minnow [27]. There are also some differences between the fathead minnow core microbiome and that of other related fishes. For example, the phylum Firmicutes occurs with notable relative abundance in some (but not all) gut communities reported for zebrafish and guppy [25], but is not present in the fathead minnow core microbiome or abundant at any point in the full experiment. Similarity of commensal bacterial communities among phylogenetically related and anatomically similar hosts has been shown for non-fish vertebrate species [81] and suggested for fish [77]. Our study adds to the body of evidence that gut microbial community structure may also be conserved among phylogenetically related fishes.
The gut microbial community of individual fish is likely to be most strongly structured by the interacting effects of 1) host environment and diet, 2) host developmental stage, and 3) triclosan exposure history. The baseline communities were markedly different than days 7 to 21 , and this difference made a strong contribution to the overall betadiversity temporal trajectory (Figure 3a). While almost all OTUs present at days 7 to 21 are also present in the baseline (day 0) samples, because we did not sequence samples of water (autoclaved) or food (commercially prepared), we cannot definitively rule out water or food as a source of the few new OTUs we detected. All exposure groups received food and water from identical stock at each feeding or water change, so any new OTUs that might have arisen from these sources are expected to be evenly distributed across cohorts. The baseline samples were collected immediately upon arrival in our facility, and differing community structure more likely reflects differing conditions in the rearing facility from which the fish were acquired, as diet, water chemistry, and stress have been suggested to be predictive of fish gut microbiome structure $[24,25,77,80]$. However, even when the highly divergent baseline samples were removed from consideration, communities were in large part structured by time point/developmental stage (Figure $3 \mathrm{~b}$ ). Our study was designed to examine the effect of short-term triclosan exposure on the endogenous gut microbiota of developing, larval fish. Gut microbiomes of developing vertebrates, including fish, are dynamic, showing complex successional processes [82-85]. Thus, the temporally dynamic communities observed here, in addition to effects of triclosan exposure, likely respond to the combined effects of host developmental processes and initial changes to environment.

\section{Conclusions}

Most research on the impact of environmental contaminants has focused on aquatic animals and invertebrates [86] or on water, sediment, and soil microbial communities [51,87]. The effects of environmental contaminants on the host-associated microbiome are largely unexplored. This study demonstrates a shift in the fish gut bacterial community following a 7-day exposure to low, environmentally relevant levels of triclosan. Taxa whose relative abundances change with triclosan exposure include those potentially involved in nitrogen cycling and triclosan metabolism. Even short-duration disruption to the host microbiome such as that shown here may induce longterm effects on the host organism and larger ecosystem.

\section{Methods}

\section{Experimental design}

The effects of acute early-life stage exposure to environmentally relevant concentrations of triclosan on the composition of the gastrointestinal tract microbiome in 
larval fathead minnow (P. promelas) were evaluated in an immersion exposure experiment. Fathead minnow larvae (approximately 8 weeks post-hatch) were obtained from Aquatic Biosystems (Fort Collins, CO). At this developmental stage, fish were sexually undifferentiated or were undergoing differentiation and gender was not determined. The rearing facility classifies fish by hatch age, and the fish in this study were all hatched within a 4-day period. The fish are not isogenic, rather are the progeny of multiple breeding groups. Thus, sibship and age are untested variables in our study.

Eight randomly selected fish were sampled upon arrival as initial controls (baseline; day 0) as described below. The remaining fish were randomly assigned to experimental and control groups. For each group, 15 larvae were placed into each of two 4-L glass jars containing $1 \mathrm{~L}$ of solution. All glassware and aeration tubing was autoclaved prior to use, and food was introduced using sterile, single-use serological pipettes. A stock solution of sterile hatched brine shrimp (Hikari Bio-Pure Baby Brine Shrimp, Hayward CA) was prepared daily, and larvae were presented with $2 \mathrm{~mL}$ of this stock solution and allowed to feed ad libitum. We did not reverify food sterility in-house or sequence a food-only sample, and thus cannot rule out the possibility that food contributed OTUs to our study; however, at each feeding, all fish were fed from the same stock solution. Our design and methods do not differentiate between transient (including any food-associated) bacteria and adherent bacteria. Test solutions for the 7-day acute exposure were a) control: moderately hard reconstituted water (MHRW) as defined by US Environmental Protection Agency (EPA) protocol \#EPA-821-R-02-013 [88], b) solvent control: $0.0001 \mathrm{mg} \mathrm{L}^{-1}$ methanol in MHRW, c) low triclosan: $100 \mathrm{ng} \mathrm{L}^{-1}$ triclosan in MHRW, and d) high triclosan: 1,000 ng L ${ }^{-1}$ in MHRW. Triclosan (SigmaAldrich) was solubilized in methanol, and methanol concentrations were identical across solvent control and triclosan solutions. Methanol was chosen as the solvent for consistency with a recent study focusing on the direct physiological effects of triclosan on the fathead minnow [17]. MHRW involves autoclaved $\left(121^{\circ} \mathrm{C}, 15 \mathrm{psi}, 30 \mathrm{~min}\right)$, sterilized water in addition to the additives described in EPA protocol \#EPA-821-R-02-013. Our measured pH and alkalinity align with those of similar husbandry schemes and experimental designs, including those of the breeding facility. To utilize environmentally relevant concentrations of triclosan, we chose 100 and 1,000 ng/L which allowed us to work within the Kolpin et al. study median of 140 and max of 2,300 ng/L [15], while still working below the reported LC50 for $P$. promelas $(260 \mu \mathrm{g} / \mathrm{L}$ at 96-h duration) [89].

Over a 7 -day acute exposure, $>90 \%$ of the test solution was replaced daily. Daily static renewal exchanges consisted of $100 \mu \mathrm{L}$ aliquots of the pertinent spike aliquot dissolved into $1 \mathrm{~L}$ of MHRW. At each daily renewal, every cohort was moved into a freshly autoclaved jar. Following the 7-day acute exposure, larvae from all exposure groups were maintained in control conditions with daily renewals into MHRW and fresh jars until the end of the experiment on day 21 (Figure 1). Photoperiod (14 h light: $10 \mathrm{~h}$ dark), temperature $\left(22 \pm 1^{\circ} \mathrm{C}\right)$, and dissolved oxygen ( $>85 \%$ saturation) did not differ among exposure groups during the 21-day experiment. Animal care and handling was in accordance with the Institutional Animal Care and Use Committee of the University of Colorado Denver, \#92514(05)1E.

\section{Sample collection}

Multiple intact gastrointestinal (GI) tracts were collected at four time points for each experimental group: prior to exposure (baseline), immediately after a 7-day exposure (day 7), after 1 week of post-exposure depuration (day 14 ), and finally at the end of the 21-day experiment (day 21 ). Fish were anesthetized in ice water prior to euthanasia by rapid decapitation. Body length and mass measurements of anesthetized fish were collected and are included in Additional file 1: Table S1. Freshly dissected GI tracts were placed into filter-sterilized PBS and frozen at $-20^{\circ} \mathrm{C}$ until DNA extraction. Since the gastrointestinal organ is less developed in larval fish compared to adult fish, the transition between esophagus, stomach, and intestines was not distinguishable during dissection. Manipulation instruments were autoclaved, and instruments and surfaces were cleaned with ethanol after each specimen. All work was performed in a biosafety cabinet.

\section{DNA extraction}

Total bacterial and host DNA was extracted using the PowerSoil DNA Isolation Kit (MO BIO Laboratories, Carlsbad CA), with modifications to the standard protocol noted below. Samples were thawed and transferred with $50 \mu \mathrm{L}$ of PBS storage buffer to the bead tube, which was then vortexed at maximum speed for $10 \mathrm{~min}$ to disrupt the intact fish GI tracts. After the addition of solution $\mathrm{C} 1$, the tube was heated at $65^{\circ} \mathrm{C}$ for $10 \mathrm{~min}$, followed by $10 \mathrm{~min}$ of vortexing at maximum speed. Following the $\mathrm{C} 3$ incubation step, the centrifugation time was increased from 1 to $2 \mathrm{~min}$. Extracted DNA was stored at $-20^{\circ} \mathrm{C}$. Because these extractions contained varying, unknown ratios of host to microbial gDNA, we used the spectroscopic DNA quantification as rough estimates of extraction success, but were unable to use these measurements for normalization of microbial gDNA mass for downstream processing. 


\section{Marker gene (16S rDNA) amplification and sequencing}

Amplicon sequencing of 108 samples targeting the V3V4 hypervariable regions of the $16 \mathrm{~S}$ rRNA gene was performed following the dual-indexing strategy of Kozich et al. [90]. The 103 experimental samples included eight baseline samples and eight samples per triclosan exposure group for each of the three subsequent time-points (Figure 1), with the exception of 'day 14 low' which had only seven samples due to the failure of one sample in DNA extraction. In addition to these 103 samples, we sequenced one technical replicate of sample D0C9, one sample of a mock bacterial community consisting of equal mass of gDNA from ten different bacterial and archaeal species [91] (Table 1), and three mock/spike technical replicates containing a 1:1 combination (by gDNA mass) of the mock community and sample D0C9. D0C9 was chosen because sufficient gDNA was available, and a pilot study suggested that this sample was representative of other baseline samples. PCR reactions included $10 \mu \mathrm{L}$ Q5 $2 \times$ Hot Start Master Mix (New England Biolabs, Ipswich MA), $0.5 \mu \mathrm{L}$ each primer (from $10 \mu \mathrm{M}$ stock), $1 \mu \mathrm{L}$ template DNA, and $8 \mu \mathrm{L}$ nuclease-free water under the following conditions: $30 \mathrm{~s}$ at $98^{\circ} \mathrm{C} ; 25$ cycles of $\left(10 \mathrm{~s}\right.$ at $98^{\circ} \mathrm{C}$, $15 \mathrm{~s}$ at $55^{\circ} \mathrm{C}, 20 \mathrm{~s}$ at $\left.72^{\circ} \mathrm{C}\right) ; 2 \mathrm{~min}$ at $72^{\circ} \mathrm{C} ; 4^{\circ} \mathrm{C}$ hold. Triplicate reactions per sample were combined and cleaned up using the Zymo Clean and Concentrate-5 kit (Zymo Research Corporation, Irvine CA), eluted into $17 \mu \mathrm{L}$ nuclease-free water and quantified using the Qubit BR dsDNA kit (Life Technologies, Grand Island NY). Additional PCR reactions were performed as needed to generate the $10 \mathrm{ng}$ of cleaned amplicon from each sample included in the pooled sequencing library. Sequencing was performed at the University of Colorado Denver Genomics and Microarray Core with a single lane of Illumina MiSeq using $2 \times 251$ bp paired end reads and V2 chemistry, with $8 \%$ PhiX added to the library. All reads and metadata are deposited in the Sequence Read Archive (SRA; http://www.ncbi.nlm.nih.gov/sra) under BioProject PRJNA257816 (SRA accession SRP045371).

\section{Read preprocessing, OTU assignment, and OTU filtering}

Demultiplexing was performed with CASAVA v. 1.8, and reads representing the PhiX or reads not matching indices were removed. The remaining reads were assigned to OTUs following the UPARSE pipeline (usearch v7.0.1090_ i86linux32) [92] with the following non-default parameters: -fastq_mergepairs (-fastq_truncqual 3, -fastq_ minmergelen 250); -fastq_filter (-fastq_maxee 1.0, -fastq truncqual 10, -fastq_minlen 300); -usearch_global (-strand plus, -id 0.97) and custom scripting to accommodate large file sizes. The uchime_ref step was omitted, and the final OTUs were checked for chimeras using the DECIPHER web tool and the short sequences option [93]. Taxonomy was assigned to OTUs in QIIME $\mathrm{v}$ 1.8.0 [94], using the
RDP classifier v2.2 [95] which was retrained against the Greengenes 13_8 rep set [33] that had been trimmed using PrimerProspector v. 1.0.1 [96] to the V3-V4 region [97]. A phylogenetic tree of the OTU sequences was constructed using FastTree [98].

We used the mock community sample to determine a threshold for filtering our OTU table for spurious or contaminant OTUs. Of the mock community reads, 99.89\% were assigned to OTUs representing mock community 16S rRNA sequences. All OTUs in the remaining $0.11 \%$ coincided with sequences found in the fish samples, and the most abundant non-mock sequence seen in the mock community sample constituted $0.03 \%$ of the reads. We used this value to filter the whole-study OTU table according to the following criteria. To be retained for further analysis, an OTU should be found at greater than $0.03 \%$ relative abundance in at least $N-3$ samples where $N$ is the size of a time + exposure group (e.g., day 7, solvent: $N=8$ ). This procedure should eliminate or greatly reduce instances of PCR contamination between samples, as we do not expect contamination to occur in a biologically meaningful pattern consistent with our experimental design. Note that OTUs can still occur at $<0.03 \%$ relative abundance in any given sample. This conservative filtering procedure runs the risk of excluding naturally occurring, low abundance OTUs near the detection limits of the filtering protocol; however, this is not expected to impact our ability to answer the diversity-based question posed in this study regarding the effects of triclosan exposure. We explicitly tested this by using a variety of beta diversity distance metrics on the filtered and unfiltered OTU tables and by calculating alpha diversity on the filtered and unfiltered OTU tables. All analyses presented here are conducted on the filtered OTU table unless otherwise noted, with no additional normalizations or rarefying procedures [99]. Both filtered and unfiltered OTU tables (Additional files 13 and 14) and OTU FASTA files (Additional files 15 and 16) are available.

\section{CK-1C4-19 phylogeny}

Publicly available sequences classified as belonging to the taxon CK-1C4-19 were downloaded from the Silva rRNA database SSU Ref web release 117 [34]. The V3V4 region was extracted in silico from these 124 sequences using PrimerProspector v. 1.0.1 [96]. Of the 124 sequences, 62 contained priming sites deemed likely to successfully amplify. Examination of sequences predicted not to amplify revealed many misclassified eukaryotes or sequences with low pintail scores, which may be chimeras. The remaining 62 sequences were combined with CK-1C4-19 sequences from this study and several Aeromonas sequences as an outgroup, aligned using MUSCLE v3.8.31 with default parameters [100], and an approximately- 
maximum-likelihood phylogenetic tree generated using FastTree 2.1.5 SSE3 [98].

\section{Statistical analyses}

Data visualization and statistical analyses were conducting using QIIME, R (http://www.r-project.org), phyloseq [101], vegan [102], and ggplot2 [103]. Generation of taxa summary bar charts and core microbiome calculation was performed using QIIME v. 1.8.0. All distance measures and ordinations were calculated in R v. 3.1.0 using phyloseq v. 1.8.2, and vegan v. 2.0-10. Shannon's diversity index was calculated using phyloseq, with ANOVA and Tukey's HSD tests performed in R. We used PERMANOVA (vegan::adonis) [29] with the weighted UniFrac distance matrix in order to test the ability of multiple variables (time, triclosan exposure) to account for observed variance in inter-sample distances. To calculate the significance of clusters observed in principle coordinates analysis, we used MRPP (vegan::mrpp), a univariate analysis that compares mean within-group distance against the withingroup distance of randomly permuted groups [31]. MRPP was performed with 10,000 permutations on a pairwise weighted UniFrac distance matrix for all samples within each time point, with groups defined by triclosan exposure category. To identify differentially abundant OTUs, we used the DESeq2 [32] package for $\mathrm{R}$ which has been extended to the analysis of microbial community data via phyloseq [99] with parameters: test $=$ Ward, fit $=$ local, $P \leq 0.005$.

\section{Availability of supporting data}

The data sets supporting the results of this article are available in the NCBI Sequence Read Archive, BioProject PRJNA257816, SRA accession SRP045371 (http://www. ncbi.nlm.nih.gov/sra).

\section{Additional files}

Additional file 1: Table S1. Sample identification codes, BioSample accession numbers, experiment information, barcoding indices, and number of reads for each of the 108 characterized microbiomes.

Additional file 2: Figure S2. Overall patterns of beta-diversity are unaffected by OTU filtering procedure. Principle coordinates analysis of weighted UniFrac inter-sample distances using the unfiltered OTU table differs little from results shown in Figure 3, with samples grouped primarily by time point.

Additional file 3: Figure S3. Samples cluster by exposure level immediately following acute triclosan exposure when using the unfiltered OTU table for ordination. Analyses are as in Figure 4. MRPP values: day $7(A=0.195, P<0.0001)$; day $14(A=0.071, P=0.022)$; day 21 $(A=0.003, P=0.377)$.

Additional file 4: Figure S4. Amplicon sequencing of the $16 \mathrm{~S}$ V3-V4 region is technically reproducible, and filtering removes spurious OTUs. a) Sample D0C9 and its technical replicate are highly similar both pre-filtering for spurious OTUs (Pearson $r=0.99$ ) and post-filtering (Pearson $r=0.99$ ). b) One mock community sample was prepared and sequenced. Levels of non-mock community OTUs (e.g., from crosscontamination from other fish gut samples) in the mock community sample were used to set a study-wide minimum abundance threshold ( $\geq 0.03 \%)$ for inclusion. In the full experiment, if an OTU reached this $0.03 \%$ threshold in $\geq 5$ of eight samples in any time + exposure group, it was retained for analyses. c) Three spike-in samples were prepared with equal parts mock community gDNA and sample DOC9 gDNA (mixed fish and bacterial community). Because fish gDNA dominates the DoC9 component; the bacterial community identified in pre-filtering spike-in samples is dominated by the mock community, as expected. d) After identifying spurious OTUs from the study-wide filtering, all OTUs from mock community members are successfully removed from the spike-in samples, leaving only OTUs from the DOC9 component. The post-filtering spike-in samples (mean remaining reads per sample $=263$ ) correctly resemble the D0C9 sample (mean pairwise $r=0.85$ ). Each color represents a different genus, with taxonomy presented for selected taxa as assigned via the RDP classifier and the Greengenes database (see the 'Methods' section).

Additional file 5: Figure S5. Relative abundances for the fathead minnow core microbiome OTUs across individual fish. OTUs identified as part of the core microbiome (present across at least 95\% of the 103 sample fish) were clustered and ordered by single-linkage hierarchical clustering based on Euclidean distances of day 7 cells. White cells in the heat map indicate no reads assigned to the OTU for that sample. Relative abundances are normalized to the total number of reads per sample, and scale bar (fractional relative abundance) and coloring is identical to Figure 5. Taxonomic assignments of core OTUs are available in Table 2. Columns are B: baseline, C: Control, S: Solvent, L: Low triclosan exposure history, and $\mathrm{H}$ : High triclosan exposure history.

Additional file 6: Figure S6. Alternate distance and ordination methods do not alter overall study-wide patterns of beta diversity. a) Principle coordinates ordination of Canberra distances for all samples. b) Principle coordinates ordination of Canberra distances with Baseline samples removed. c) NMDS ordination of Morisita distances for all samples. d) NMDS ordination of Morisita distances with Baseline samples removed.

Additional file 7: Figure S1. Genus-level diversity presented as relativeabundances per sample of each assigned genus, across all 108 samples referenced in this study. Each color in the stacked bar chart represents a genus. Sample codes are as in Table S1.

Additional file 8: Figure S7. Samples cluster by exposure level immediately following acute triclosan exposure when using multiple distance metrics and ordination methods. a to c) Principle coordinates analysis of Canberra distances. MRPP values: day $7(A=0.0 .095, P=0.001)$; day $14(A=0.025, P=0.032)$; day $21(A=0.011, P=0.124) ; d$ to f) NMDS ordination of Morisita distances. MRPP values: day $7(A=0.0 .095, P=0.001)$; day $14(A=0.025, P=0.032)$; day $21(A=0.011, P=0.124)$. Analyses are otherwise as in Figure 4, and use the filtered OTU table.

Additional file 9: Table S2. DESeq2 results identifying differentially abundant OTUs by triclosan exposure history (high/solvent vs. control/ solvent). Twenty-eight OTUs are differentially abundant at day 7, 2 OTUs are differentially abundant at day 14 , and no OTUs are differentially abundant at day 21.

Additional file 10: Table S3. DESeq2 results identifying differentially abundant OTUs by high triclosan exposure history with respect to methanol solvent-exposed samples (high vs. solvent). Nine OTUs are differentially abundant at day 7, five OTUs are differentially abundant at day 14, and six OTUs are differentially abundant at day 21.

Additional file 11: Table S4. Significant pairwise comparisons for OTUs shown in Figure 7.

Additional file 12: Figure S8. Abundance patterns of individual OTUs may be obscured by higher-level taxonomic assignment. a) Relative abundance of ten OTUs within order Pseudomonadales, half of which are assigned as genus Acinetobacter. b to e) Relative abundance of the four Acinetobacter OTUs identified as significantly increased in triclosanexposed samples at day 7. f) Acinetobacter OTU34 is not significantly increased in triclosan-exposed at day 7.

Additional file 13: Filtered OTU table. Read counts per sample for OTUs passing the filtering procedure, in tab-delimited text format.

Additional file 14: Unfiltered OTU table. Read counts per sample for all OTUs, including those not passing the filtering procedure, in tab-delimited text format. 
Additional file 15: FASTA file of filtered OTU sequences. FASTA formatted text file of DNA sequences for all OTUs passing the filtering procedure.

Additional file 16: FASTA file of unfiltered OTU sequences. FASTA formatted text file of DNA sequences for all OTUs, including those not passing the filtering procedure.

\section{Competing interests}

The authors declare that they have no competing interests.

\section{Authors' contributions}

ABN, MAL, TMR, AMV, and CSM conceived the study. MAL, AMV, ABN, and TMR performed the exposure experiment and sample collection. ABN, EPS, and KJB carried out the molecular biology experiments. ABN and CSM performed the computational work. ABN, TMR, and CSM analyzed the data. ABN, CSM, MAL, and AMV wrote the manuscript. All authors read, edited, and approved the final manuscript.

\section{Acknowledgements}

This work was funded by the University of Colorado Denver, College of Liberal Arts and Sciences. We thank Aaron N. Johnson for the microdissection assistance and advice. We thank Michael Wunder for the advice on statistical reporting. We thank Angelina Baroffio for the assistance with dissections and fish husbandry. We thank Katrina Diener, Ted Shade, and the University of Colorado Denver Genomics and Microarray Core for the sequencing and informatics assistance. MAL is supported by funding from the Saudi Arabian Cultural Mission.

\section{Author details}

'Department of Integrative Biology, University of Colorado Denver, Campus Box 171, PO Box 173364, Denver, CO 80217, USA. ${ }^{2}$ Paul G. Allen School for Global Animal Health, College of Veterinary Medicine, Washington State University, Pullman, WA 99164, USA. ${ }^{3}$ School of Molecular Biosciences, College of Veterinary Medicine, Washington State University, Pullman, WA 99164, USA

Received: 9 August 2014 Accepted: 29 January 2015

Published online: 03 March 2015

\section{References}

1. Relman DA. The human microbiome: ecosystem resilience and health. Nut Rev. 2012:70 Suppl 1:S2-9.

2. Huttenhower C, Gevers D, Knight R, Abubucker S, Badger JH, Chinwalla AT, et al. Structure, function and diversity of the healthy human microbiome. Nature. 2012;486:207-14.

3. Kovatcheva-Datchary P, Tremaroli V, Bäckhed F. The gut microbiota. In: Rosenberg E, DeLong EF, Lory S, Stackebrandt E, Thompson F, editors. The prokaryotes. Berlin, Heidelberg: Springer Berlin Heidelberg; 2013. p. 3-24.

4. Faith JJ, Guruge JL, Charbonneau M, Subramanian S, Seedorf H, Goodman $\mathrm{AL}$, et al. The long-term stability of the human gut microbiota. Science. 2013:341:1237439.

5. Dethlefsen $L$, Relman DA. Incomplete recovery and individualized responses of the human distal gut microbiota to repeated antibiotic perturbation. Proc Natl Acad Sci U S A. 2011;108(Suppl):4554-61.

6. Cho I, Yamanishi S, Cox L, Methé BA, Zavadil J, Li K, et al. Antibiotics in early life alter the murine colonic microbiome and adiposity. Nature. 2012;488:621-6.

7. Lozupone CA, Stombaugh J, Gordon Jl, Jansson JK, Knight R. Diversity, stability and resilience of the human gut microbiota. Nature. 2012;489:220-30.

8. Jernberg C, Löfmark S, Edlund C, Jansson JK. Long-term impacts of antibiotic exposure on the human intestinal microbiota. Microbiology. 2010;156(Pt 11):3216-23.

9. Pérez-Cobas AE, Gosalbes MJ, Friedrichs A, Knecht H, Artacho A, Eismann K et al. Gut microbiota disturbance during antibiotic therapy: a multi-omic approach. Gut. 2013;62:1591-601.

10. Kraglund F. Triclosan produces statistically significant reduction in plaque, gingivitis and caries but not clinically important benefit. Evid Based Dent. 2014;15:6-7.

11. Syed AK, Ghosh S, Love NG, Boles BR. Triclosan promotes Staphylococcus aureus nasal colonization. MBio. 2014;5:e01015.
12. Halden RU. On the need and speed of regulating triclosan and triclocarban in the United States. Environ Sci Technol. 2014:48:3603-11.

13. Dann AB, Hontela A. Triclosan: environmental exposure, toxicity and mechanisms of action. J Appl Toxicol. 2011:31:285-311.

14. Heidler J, Halden RU. Mass balance assessment of triclosan removal during conventional sewage treatment. Chemosphere. 2007;66:362-9.

15. Kolpin DW, Furlong ET, Meyer MT, Thurman EM, Zaugg SD, Barber LB, et al. Pharmaceuticals, hormones, and other organic wastewater contaminants in U.S. streams, 1999-2000: a national reconnaissance. Environ Sci Technol. 2002;36(6):1202-11.

16. Lankester J, Patel C, Cullen MR, Ley C, Parsonnet J. Urinary triclosan is associated with elevated body mass index in NHANES. PLoS One. 2013;8:e80057.

17. Fritsch EB, Connon RE, Werner I, Davies RE, Beggel S, Feng W, et al. Triclosan impairs swimming behavior and alters expression of excitation-contraction coupling proteins in fathead minnow (Pimephales promelas). Environ Sci Technol. 2013;47:2008-17.

18. Cherednichenko G, Zhang R, Bannister RA, Timofeyev V, Li N, Fritsch EB, et al. Triclosan impairs excitation-contraction coupling and Ca2+dynamics in striated muscle. Proc Natl Acad Sci U S A. 2012;109:14158-63.

19. Raut SA, Angus RA. Triclosan has endocrine-disrupting effects in male western mosquitofish, Gambusia affinis. Environ Toxicol Chem. 2010:29:1287-91.

20. Kuehn BM. FDA pushes makers of antimicrobial soap to prove safety and effectiveness. JAMA. 2014;311:234

21. Marty J, Hawj F, Hoffman JA, Eaton CA, Scalze B, Hortman M, et al. SF2192/ HF2452. Minnesota State Legislature. 2014;277:10841.

22. Ankley GT, Villeneuve DL. The fathead minnow in aquatic toxicology: past, present and future. Aquat Toxicol. 2006;78:91-102.

23. Roeselers G, Mittge EK, Stephens WZ, Parichy DM, Cavanaugh CM, Guillemin K, et al. Evidence for a core gut microbiota in the zebrafish. ISME J. 2011:5:1595-608.

24. Nayak SK. Role of gastrointestinal microbiota in fish. Aquac Res. 2010;41:1553-73.

25. Sullam KE, Essinger SD, Lozupone CA, O'Connor MP, Rosen GL, Knight R, et al. Environmental and ecological factors that shape the gut bacterial communities of fish: a meta-analysis. Mol Ecol. 2012;21:3363-78.

26. Wong S, Waldrop T, Summerfelt S, Davidson J, Barrows F, Kenney PB, et al. Aquacultured rainbow trout (Oncorhynchus mykiss) possess a large core intestinal microbiota that is resistant to variation in diet and rearing density. Appl Environ Microbiol. 2013;79:4974-84.

27. Lochmann R, Phillips H, Xie L. Effects of a dairy-yeast prebiotic and water hardness on the growth performance, mineral composition and gut microflora of fathead minnow (Pimephales promelas) in recirculating systems. Aquaculture. 2011;320:76-81.

28. Lozupone C, Lladser ME, Knights D, Stombaugh J, Knight R. UniFrac: an effective distance metric for microbial community comparison. ISME J. 2011:5:169-72.

29. Anderson MJ. A new method for non-parametric multivariate analysis of variance. Austral Ecol. 2001:26:32-46.

30. Kuczynski J, Liu Z, Lozupone C, McDonald D, Fierer N, Knight R. Microbial community resemblance methods differ in their ability to detect biologically relevant patterns. Nat Methods. 2010;7:813-9.

31. Mielke PW, Berry KJ, Johnson ES. Multi-response permutation procedures for a priori classifications. Commun Stat - Theor Meth. 1976;A5:1409-24.

32. Love MI, Huber W, Anders S. Moderated estimation of fold change and dispersion for RNA-seq data with DESeq2. 2014.

33. DeSantis TZ, Hugenholtz P, Larsen N, Rojas M, Brodie EL, Keller K, et al. Greengenes, a chimera-checked 16S rRNA gene database and workbench compatible with ARB. Appl Environ Microbiol. 2006;72:5069-72.

34. Quast C, Pruesse E, Yilmaz P, Gerken J, Schweer T, Yarza P, et al. The SILVA ribosomal RNA gene database project: Improved data processing and web-based tools. Nucleic Acids Res. 2013;41:D590-6.

35. McMurry LM, Oethinger M, Levy SB. Triclosan targets lipid synthesis. Nature. 1998;394:531-2.

36. Heath RJ, Rubin JR, Holland DR, Zhang E, Snow ME, Rock CO. Mechanism of triclosan inhibition of bacterial fatty acid synthesis. J Biol Chem. 1999;274:11110-4

37. Heath RJ, Rock CO. A triclosan-resistant bacterial enzyme. Nature. 2000;406:145-6.

38. Heath RJ, Su N, Murphy CK, Rock CO. The enoyl-[acyl-carrier-protein] reductases Fabl and FabL from Bacillus subtilis. J Biol Chem. 2000;275:40128-33.

39. Massengo-Tiassé RP, Cronan JE. Vibrio cholerae FabV defines a new class of enoyl-acyl carrier protein reductase. J Biol Chem. 2008;283:1308-16. 
40. Chuanchuen R, Karkhoff-Schweizer RR, Schweizer HP. High-level triclosan resistance in Pseudomonas aeruginosa is solely a result of efflux. Am J Infect Control. 2003;31:124-7

41. Russell AD. Whither triclosan? J Antimicrob Chemother. 2004;53:693-5.

42. Decostere A. Flavobacterium columnare infections in fish: the agent and its adhesion to the gill tissue. Verh K Acad Geneeskd Belg. 2002;64:421-30.

43. Zamora L, Vela Al, Palacios MA, Domínguez L, Fernández-Garayzábal JF. First isolation and characterization of Chryseobacterium shigense from rainbow trout. BMC Vet Res. 2012;8:77.

44. Janda JM, Abbott SL. The genus Shewanella: from the briny depths below to human pathogen. Crit Rev Microbiol. 2014;40:293-312.

45. Vignier N, Barreau M, Olive C, Baubion E, Théodose R, Hochedez P, et al. Human infection with Shewanella putrefaciens and S. algae: report of 16 cases in Martinique and review of the literature. Am J Trop Med Hyg. 2013;89:151-6.

46. Hoshino T, Terahara T, Tsuneda S, Hirata A, Inamori Y. Molecular analysis of microbial population transition associated with the start of denitrification in a wastewater treatment process. J Appl Microbiol. 2005;99:1165-75.

47. Etchebehere C, Errazquin I, Barrandeguy E, Dabert P, Moletta R, Muxí L. Evaluation of the denitrifying microbiota of anoxic reactors. FEMS Microbiol Ecol. 2001;35:259-65.

48. Lu H, Chandran K, Stensel D. Microbial ecology of denitrification in biological wastewater treatment. Water Res. 2014;64:237-54

49. Liu B, Zhang F, Feng $X$, Liu $Y$, Yan $X$, Zhang $X$, et al. Thauera and Azoarcus as functionally important genera in a denitrifying quinoline-removal bioreactor as revealed by microbial community structure comparison. FEMS Microbiol Ecol. 2006;55:274-86

50. Hallin S, Throbäck IN, Dicksved J, Pell M. Metabolic profiles and genetic diversity of denitrifying communities in activated sludge after addition of methanol or ethanol. Appl Environ Microbiol. 2006;72:5445-52.

51. Drury B, Scott J, Rosi-Marshall EJ, Kelly JJ. Triclosan exposure increases triclosan resistance and influences taxonomic composition of benthic bacterial communities. Environ Sci Technol. 2013:47:8923-30.

52. Walke JB, Becker MH, Loftus SC, House LL, Cormier G, Jensen RV, et al. Amphibian skin may select for rare environmental microbes. ISME J. 2014;8:2207-17.

53. Kormas KA, Meziti A, Mente E, Frentzos A. Dietary differences are reflected on the gut prokaryotic community structure of wild and commercially reared sea bream (Sparus aurata). Microbiologyopen. 2014;3:718-28.

54. Van Kessel MA, Dutilh BE, Neveling K, Kwint MP, Veltman JA, Flik G, et al. Pyrosequencing of $16 \mathrm{~S}$ rRNA gene amplicons to study the microbiota in the gastrointestinal tract of carp (Cyprinus carpio L.). AMB Express. 2011;1:41.

55. Stief $P$, Poulsen $M$, Nielsen LP, Brix H, Schramm A. Nitrous oxide emission by aquatic macrofauna. Proc Natl Acad Sci U S A. 2009;106:4296-300.

56. Wüst PK, Horn MA, Henderson G, Janssen PH, Rehm BHA, Drake HL. Gutassociated denitrification and in vivo emission of nitrous oxide by the earthworm families Megascolecidae and Lumbricidae in New Zealand. Appl Environ Microbiol. 2009;75:3430-6

57. Davidson SK, Stahl DA. Selective recruitment of bacteria during embryogenesis of an earthworm. ISME J. 2008;2:510-8.

58. Lundberg JO, Weitzberg E, Cole JA, Benjamin N. Nitrate, bacteria and human health. Nat Rev Microbiol. 2004;2:593-602.

59. Meyer F, Trimble W, Chang E, Handley K. Functional predictions from inference and observation in sequence-based inflammatory bowel disease research. Genome Biol. 2012:13:169.

60. Mechichi T, Stackebrandt E, Gad'on N, Fuchs G. Phylogenetic and metabolic diversity of bacteria degrading aromatic compounds under denitrifying conditions, and description of Thauera phenylacetica sp. nov., Thauera aminoaromaticasp. nov., and Azoarcus buckelii sp. nov. Arch Microbiol. 2002;178:26-35.

61. Monferrán MV, Echenique JR, Wunderlin DA. Degradation of chlorobenzenes by a strain of Acidovorax avenae isolated from a polluted aquifer. Chemosphere. 2005;61:98-106.

62. Lambo AJ, Patel TR. Biodegradation of polychlorinated biphenyls in Aroclor 1232 and production of metabolites from 2,4,4'-trichlorobiphenyl at low temperature by psychrotolerant Hydrogenophaga sp. strain IA3-A. J Appl Microbiol. 2007;102:1318-29.

63. Sueoka K, Satoh H, Onuki M, Mino T. Microorganisms involved in anaerobic phenol degradation in the treatment of synthetic coke-oven wastewater detected by RNA stable-isotope probing. FEMS Microbiol Lett. 2009;291:169-74.

64. Lolas IB, Chen X, Bester K, Nielsen JL. Identification of triclosan-degrading bacteria using stable isotope probing, fluorescence in situ hybridization and microautoradiography. Microbiology. 2012;158(Pt 11):2796-804.
65. Lee DG, Chu K-H. Effects of growth substrate on triclosan biodegradation potential of oxygenase-expressing bacteria. Chemosphere. 2013;93:1904-11.

66. Lee DG, Cho K-C, Chu K-H. Identification of triclosan-degrading bacteria in a triclosan enrichment culture using stable isotope probing. Biodegradation. 2014;25:55-65.

67. Ohtsubo Y, Maruyama F, Mitsui H, Nagata Y, Tsuda M. Complete genome sequence of Acidovorax sp. strain KKS102, a polychlorinated-biphenyl degrader. J Bacteriol. 2012;194:6970-1.

68. Shapleigh JP. The denitrifying prokaryotes. In: Rosenberg E, DeLong EF, Lory S, Stackebrandt E, Thompson F, editors. The prokaryotes. Berlin, Heidelberg: Springer Berlin Heidelberg; 2013. p. 405-25.

69. Leahy JG, Colwell RR. Microbial degradation of hydrocarbons in the environment. Microbiol Rev. 1990:54:305-15.

70. Balmer ME, Poiger T, Droz C, Romanin K, Bergqvist P-A, Müller MD, et al. Occurrence of methyl triclosan, a transformation product of the bactericide triclosan, in fish from various lakes in Switzerland. Environ Sci Technol. 2004;38:390-5

71. Janda JM, Abbott SL. The genus Aeromonas: taxonomy, pathogenicity, and infection. Clin Microbiol Rev. 2010;23:35-73.

72. Jung $A$, Jung-Schroers $V$. Detection of Deefgea chitinilytica in freshwater ornamental fish. Lett Appl Microbiol. 2011;52:497-500.

73. Chu W, Zhou S, Zhu W, Zhuang X. Quorum quenching bacteria Bacillus sp. QSI-1 protect zebrafish (Danio rerio) from Aeromonas hydrophila infection. Sci Rep. 2014;4:5446

74. Boutin S, Bernatchez L, Audet C, Derôme N. Network analysis highlights complex interactions between pathogen, host and commensal microbiota. PLoS One. 2013:8:e84772.

75. Li X, Yan Q, Xie S, Hu W, Yu Y, Hu Z. Gut microbiota contributes to the growth of fast-growing transgenic common carp (Cyprinus carpio L.). PLoS One. 2013;8:e64577.

76. Semova I, Carten JD, Stombaugh J, Mackey LC, Knight R, Farber SA, et al. Microbiota regulate intestinal absorption and metabolism of fatty acids in the zebrafish. Cell Host Microbe. 2012;12:277-88.

77. Clements KD, Angert ER, Montgomery WL, Choat JH. Intestinal microbiota in fishes: what's known and what's not. Mol Ecol. 2014;23:1891-8.

78. Vajda AM, Barber LB, Gray JL, Lopez EM, Bolden AM, Schoenfuss HL, et al. Demasculinization of male fish by wastewater treatment plant effluent. Aquat Toxicol. 2011;103:213-21.

79. Llewellyn MS, Boutin S, Hoseinifar SH, Derome N. Teleost microbiomes: the state of the art in their characterization, manipulation and importance in aquaculture and fisheries. Front Microbiol. 2014;1:1.

80. Wong S, Rawls JF. Intestinal microbiota composition in fishes is influenced by host ecology and environment. Mol Ecol. 2012;21:3100-2.

81. Ley RE, Lozupone CA, Hamady M, Knight R, Gordon Jl. Worlds within worlds: evolution of the vertebrate gut microbiota. Nat Rev Microbiol. 2008;6:776-88.

82. Palmer C, Bik EM, DiGiulio DB, Relman DA, Brown PO. Development of the human infant intestinal microbiota. PLoS Biol. 2007;5:1556-73.

83. Koenig JE, Spor A, Scalfone N, Fricker AD, Stombaugh J, Knight R, et al. Succession of microbial consortia in the developing infant gut microbiome. Proc Natl Acad Sci U S A. 2011;108(Suppl):4578-85.

84. Kohl KD, Cary TL, Karasov WH, Dearing MD. Restructuring of the amphibian gut microbiota through metamorphosis. Environ Microbiol Rep. 2013;5:899-903.

85. Vadstein $O$, Bergh $\varnothing$, Gatesoupe F-J, Galindo-Villegas J, Mulero V, Picchietti S, et al. Microbiology and immunology of fish larvae. Rev Aquac. 2013;5:S1-25.

86. Chalew TE, Halden RU. Environmental exposure of aquatic and terrestrial biota to triclosan and triclocarban. J Am Water Works Assoc. 2009:45:4-13.

87. Butler E, Whelan MJ, Ritz K, Sakrabani R, van Egmond R. Effects of triclosan on soil microbial respiration. Environ Toxicol Chem. 2011;30:360-6.

88. United States Environmental Protection Agency. Short-term methods for estimating the chronic toxicity of effluents and receiving water to freshwater organisms. EPA Publication No. 821-R-02-013. 4th ed. Washington, D.C: US EPA; 2002.

89. Orvos DR, Versteeg DJ, Inauen J, Capdevielle M, Rothenstein A, Cunningham V. Aquatic toxicity of triclosan. Environ Toxicol Chem. 2002;21:1338-49.

90. Kozich JJ, Westcott SL, Baxter NT, Highlander SK, Schloss PD. Development of a dual-index sequencing strategy and curation pipeline for analyzing amplicon sequence data on the MiSeq Illumina sequencing platform. Appl Environ Microbiol. 2013;79:5112-20.

91. Morgan JL, Darling AE, Eisen JA. Metagenomic sequencing of an in vitrosimulated microbial community. PLoS One. 2010;5:e10209. 
92. Edgar RC. UPARSE: highly accurate OTU sequences from microbial amplicon reads. Nat Methods. 2013;10:996-8.

93. Wright ES, Yilmaz LS, Noguera DR. DECIPHER, a search-based approach to chimera identification for $16 \mathrm{~S}$ rRNA sequences. Appl Environ Microbiol. 2012;78:717-25.

94. Caporaso JG, Kuczynski J, Stombaugh J, Bittinger K, Bushman FD, Costello EK, et al. QIIME allows analysis of high-throughput community sequencing data. Nat Methods. 2010;7:335-6.

95. Wang Q, Garrity GM, Tiedje JM, Cole JR. Naive Bayesian classifier for rapid assignment of rRNA sequences into the new bacterial taxonomy. Appl Environ Microbiol. 2007;73:5261-7.

96. Walters WA, Caporaso JG, Lauber CL, Berg-Lyons D, Fierer N, Knight R. PrimerProspector: de novo design and taxonomic analysis of barcoded polymerase chain reaction primers. Bioinformatics. 2011;27:1159-61.

97. Werner JJ, Koren O, Hugenholtz P, DeSantis TZ, Walters WA, Caporaso JG, et al. Impact of training sets on classification of high-throughput bacterial 16 s rRNA gene surveys. ISME J. 2012;6:94-103.

98. Price MN, Dehal PS, Arkin AP. FastTree 2 - approximately maximumlikelihood trees for large alignments. PLoS One. 2010;5:e9490.

99. McMurdie PJ, Holmes S. Waste not, want not: why rarefying microbiome data is inadmissible. PLoS Comput Biol. 2014;10:e1003531.

100. Edgar RC. MUSCLE: multiple sequence alignment with high accuracy and high throughput. Nucleic Acids Res. 2004;32:1792-7.

101. McMurdie PJ, Holmes S. phyloseq: an R package for reproducible interactive analysis and graphics of microbiome census data. PLoS One. 2013:8:e61217.

102. Oksanen J, Blanchet FG, Kindt R, Legendre P, Minchin PR, O'Hara RB, et al. Package vegan. R Packag ver. 2013;254:20-8.

103. Wickham H. Elegant graphics for data analysis, vol. 35. New York: Springer 2009. p. 211

\section{Submit your next manuscript to BioMed Central and take full advantage of:}

- Convenient online submission

- Thorough peer review

- No space constraints or color figure charges

- Immediate publication on acceptance

- Inclusion in PubMed, CAS, Scopus and Google Scholar

- Research which is freely available for redistribution 